\title{
Structure of the Shaker Kv channel and mechanism of slow C-type inactivation
}

Xiao-Feng $\operatorname{Tan}^{1} \dagger$, Chanhyung Bae ${ }^{1} \dagger$, Robyn $\mathrm{Stix}^{2}$, Anabel I. Fernandez ${ }^{1}$, Kate Huffer ${ }^{1}$, Tsg-Hui Chang ${ }^{1}$, Jiansen Jiang ${ }^{3}$, José D. Faraldo-Gómez ${ }^{2}$ and Kenton J. Swartz ${ }^{1}$

1 Molecular Physiology and Biophysics Section, Porter Neuroscience Research Center, National Institute of Neurological Disorders and Stroke, National Institutes of Health, Bethesda, MD 20892

2 Theoretical Molecular Biophysics Laboratory, National Heart, Lung and Blood Institute, National Institutes of Health, Bethesda, MD 20892

3 Laboratory of Membrane Proteins and Structural Biology and Biophysics Center, National Heart, Lung, and Blood Institute, National Institutes of Health, Bethesda, MD 20892

$\uparrow \quad$ contributed equally

*Corresponding author. Email: swartzk@ninds.nih.gov 


\begin{abstract}
:
Voltage-activated potassium (Kv) channels open upon membrane depolarization and proceed to spontaneously inactivate. Inactivation controls neuronal firing rates and serves as a form of shortterm memory, and is implicated in various human neurological disorders. Here, we use highresolution cryo-electron microscopy and computer simulations to determine one of the molecular mechanisms underlying this physiologically crucial process. Structures of the activated Shaker Kv channel and of its W434F mutant in lipid bilayers demonstrate that C-type inactivation entails the dilation of the ion selectivity filter, and the repositioning of neighboring residues known to be functionally critical. Microsecond-scale molecular dynamics trajectories confirm these changes inhibit rapid ion permeation through the channel. This long-sought breakthrough establishes how eukaryotic $\mathrm{K}^{+}$channels self-regulate their functional state through the plasticity of their selectivity filters.
\end{abstract}


Voltage-activated potassium $(\mathrm{Kv})$ channels open and close in response to changes in membrane voltage, serving critical functions in electrical signaling in neurons and muscle, neurotransmitter and hormone secretion, cell proliferation and migration, and ion homeostasis ( 1 , 2). Functional studies of Shaker $\mathrm{Kv}$ channels have been foundational for establishing key mechanistic principles such as voltage-sensing, pore opening and inactivation in eukaryotes $(1,3$ 5). The structure of the related Kv1.2/2.1 paddle chimera has provided a valuable framework to begin to understand these procesess $(6,7)$, but it has been challenging to capture the protein in distinct conformations, thereby limiting mechanistic insights $(8,9)$. In addition, the phenotypes of well-characterized mutants in the Shaker Kv channel do not always translate to the Kv1.2 channel (10), leaving key questions unanswered. One of these outstanding questions is what the structural mechanism underlies the so-called C-type inactivation of Shaker Kv channels (11), a process that regulates the firing of action potentials in neurons and cardiac muscle by diminishing the availability of these channels to open. This intriguing process has been implicated in a range of neurological and psychiatric disorders $(1,12-15)$, and occurs in other $\mathrm{Kv}$ channels too. For example, the human ether-a-go-go related (hERG) Kv channel also enters a C-type inactivated state rapidly after opening, which translates into unique inwardly-rectifying properties that are critical for shaping the cardiac action potential and preventing arrhythmias (16-19). Conformational changes related to $\mathrm{C}$-type inactivation also serve important roles in $\mathrm{Ca}^{2+}$-activated $\mathrm{K}^{+}$channels and K2P channels $(20,21)$.

It has long been hypothesized that C-type inactivation in Shaker Kv channels involves a conformational change in the external pore, as it is influenced by mutations in this region as well as by ion and blocker occupancy of the selectivity filter $(11,14,15,22-34)$. X-ray structures of mutants stabilizing open and inactivated states of KcsA, a prokaryotic $\mathrm{K}^{+}$selective channel that consists of a pore domain homologous to that found in $\mathrm{Kv}$ channels, support the prevailing view that slow inactivation in $\mathrm{K}^{+}$channels is caused by a collapse of the ion selectivity filter (35-37). However, the extent to which slow inactivation in KcsA informs on the mechanism of C-type inactivation in $\mathrm{Kv}$ channels remains an open question $(15,38,39)$. In the present study we report the first-known structure of the Shaker Kv channel and that of a well-characterized mutant that dramatically accelerates C-type inactivation $(23,29,31,40,41)$. Our findings reveal that C-type inactivation in Shaker involves an unanticipated conformational rearrangement of the external pore that leads to displacement of the P-loop and dilation of the ion selectivity filter. 


\section{Results}

\section{Structure of the Shaker Kv channel in lipid nanodiscs}

The Shaker Kv channel containing an mVenus-tag along with a deletion of residues 6-46 to remove fast inactivation (Shaker-IR) $(42,43)$ was expressed in mammalian cells, purified and reconstituted into lipid nanodiscs using MSP 1E3D (8) (Fig. S1), and its structure solved using cryogenic electron microscopy (cryo-EM) Fig. S2, S3 and Table S1). The structural construct is identical to that used in extensive functional studies from many laboratories $(6,11,22-25,27-29$, $31,34,40,42-46)$. We determined the structure of the transmembrane (TM) regions of Shaker-IR to $3 \AA$ resolution after focused refinement; the map was of high quality throughout, with clearly resolved densities for most side chains, making de novo atomic model building straightforward. Only two external loops within the voltage-sensing domains were unresolved (residues $\mathrm{H} 254$ to T276 and V330 to M356) (Fig. S2).

The fold of the tetrameric Shaker-IR Kv channel is remarkably similar to that of the Kv1.2/2.1 paddle chimera channel (7) (Ca RMSD 1.23Ä, TM score 0.99; Fig. S4, S5). Each subunit in Shaker-IR is comprised of six TM helices (S1-S6), with the S1-S4 helices from each subunit forming individual voltage-sensing domains and the tetrameric arrangement of S5-S6 helices forming the central pore domain (Figure 1A,B). As in the Kv1.2/2.1 channel structure, Shaker-IR displays a domain-swapped architecture with the S1-S4 voltage-sensing domains positioned near the S5-S6 pore-forming helices of the adjacent subunit (Figure 1A,B). The cryoEM map for Shaker-IR reveals clear densities corresponding to phospholipids that appear to be bound to the protein within both the external and internal leaflets of the lipid nanodisc (Figure 1C,D); many of these densities are similar to those seen in the Kv1.2/2.1 paddle chimera solved in mixed detergent/lipid micelles (7).

The internal structure of the S1-S4 voltage-sensing domain of Shaker-IR is also similar to that in the Kv1.2/2.1 chimera, and in both cases basic residues in S4 located near the middle of the TM region are stabilized by an occluded cation binding site or charge-transfer center that is comprised of a highly conserved Phe residue in the middle of the S2 helix (F290) and acidic residues nearby in S2 and S3 (E293 and D316) (47) (Figure 1E,F). Within the conserved core region of the S4 helix, four Arg residues (R362, R365, R368 and R371) are positioned external to F290 in S2, while one Lys (K374) is positioned at the same level of the center and the innermost $\operatorname{Arg}($ R377) and Lys (K380) are positioned internal to F290 (Figure 1E,F). The fact that the four 
basic residues in S4 known to carry most of the gating charge $(48,49)$ are accessible to the external solution suggests that our structure captures the voltage-sensing domains in the activated state, which for this channel is predominant at $0 \mathrm{mV}$. Accordingly, the intracellular S6 gate region (50) is open (see below), further indicating the structure represents an activated/open state.

Within the external pore, in the ion selectivity filter, backbone carbonyl oxygens line the ion permeation pathway and are positioned similarly to other $\mathrm{K}^{+}$channel structures thought to represent a conducting state (51) (Figure 2A). Strong EM densities can be clearly discerned at four positions within the filter and along its central axis (Figure 2A); these densities are consistent with those revealed in previous structural studies (51), and appear to reflect a series of ion binding sites within the filter. In summary, there is every indication that the structure of Shaker-IR we have resolved represents an activated, open and conducting conformation.

\section{Structure of the W434F mutant Shaker Kv channel in lipid nanodiscs}

The W434F mutant of Shaker-IR has been extensively characterized and shown to be effectively non-conducting because the mutation accelerates entry of channels into the C-type inactivated state upon membrane depolarization $(23,29,31,40,41)$. We introduced the W434F mutation into our structural construct, expressed and purified the protein and reconstituted it into lipid nanodiscs using the same approach as for Shaker-IR (Fig. S1). We solved the structure of the W434F mutant with an overall resolution of $2.9 \AA$ within the TM region; as for Shaker-IR, the cryo-EM map is of excellent quality throughout, enabling us to model the complete sequence of the TM region with the only exception of two external loops in the voltage-sensors (Fig. S3, S6 and Table S1). Structural alignment of the S1-S6 regions of the W434F mutant and Shaker-IR reveals their backbone folds are very similar (Fig. S7), with low Ca rmsd (1.07 $\AA$ ) and high TM score (0.9). The structures of the S1-S4 voltage-sensing domains in the two proteins are essentially indistinguishable, and in both instances the internal pores are clearly open (Figure 3; Fig. S7).

In contrast, a pronounced conformational change occurs within the external pore (Figure 2A,B; Video S1), a region where both cryo-EM maps have the highest resolution (Extended Figs, 2,6). A key interaction between D447 in the P-loop and W434 in the pore helix, observed in Shaker-IR and most other $\mathrm{K}^{+}$channels $(7,51)$, is broken in $\mathrm{W} 434 \mathrm{~F}$, resulting in a displacement of the P-loop towards the external solution by about $5 \AA$ (Figure 2A-E; Fig. S7B). At the external end of the ion selectivity filter, the sidechain of Y445 undergoes a drastic reorientation in the 
mutant, breaking off its hydrogen-bond contact with T439 in the adjacent subunit and rotating $90^{\circ}$ to reposition behind the filter of the same subunit (Figure 2A-E; Fig. S7B). M448 and T449, two critical positions for C-type inactivation $(24,27,31)$, also move large distances, with the Ca of M448 moving about $5 \AA$ from an exposed position in Shaker-IR to a buried site where it interacts with W435 in the rapidly inactivating mutant (Figure 2A-E; Fig. S7B). In contrast, both T449 and P450 flip from being partially buried in Shaker-IR to completely exposed to solvent in W434F (Figure 2B-E; Fig. S7B,C). Interestingly, the cryo-EM density map for the mutant indicates two distinct rotamers of the D447 side chain, one of which positions the acidic residue near T449 (Figure 2E; Fig.S7C). It is apparent, therefore, that there is an overall reorganization of side chain interactions behind the filter in the inactivated state, with critical residues within the P-loop between Y445 and P450 undergoing substantial displacements. Importantly, this reorganization translates into a marked expansion at the external portion of the ion selectivity filter, by about $4 \AA$ (Figure 2A-E; Figure 3A-C; Video S1). Ion densities therein are perturbed, while those at the internal end of the filter are seemingly unaltered (Figure 2A; Fig. S8). Inspection of maps and half maps with either C4 or C1 symmetry imposed shows the density within the dilatated portion of the filter is relatively weak, suggestive of occupancy by water molecules or possibly $\mathrm{K}^{+}$ions (Figure 2A; Fig. S8).

It seems apparent that the balance of ion-protein and ion-solvent interactions that controls $\mathrm{K}^{+}$permeation in the activated state is somehow perturbed by the dilation of the external portion of the filter (Figure 3A-C) and the reconfiguration of key sidechains such as D447. However, whether conduction is indeed impaired in W434F is not self-evident from the structure alone. To evaluate this question, we calculated a series of molecular dynamics (MD) trajectories specifically designed to evaluate the ion conducting properties of the pore domain in each of the two structures determined experimentally (Materials and Methods, Fig. S9). For Shaker-IR, we calculated a trajectory of $3.5 \mu \mathrm{s}$ that included a transmembrane voltage jump from 0 to $300 \mathrm{mV}$ at $\mathrm{t}=0.5 \mu \mathrm{s}$. In symmetric $300 \mathrm{mM} \mathrm{KCl}$, we observed a total of 12 complete permeation events after the voltage jump (Figure 3D,F,G). In all cases the $\mathrm{K}^{+}$ions traversed the filter in the outward direction and in a stepwise manner, transiently interacting with each of the carbonyl groups along the way (Movie S2). One occupancy state however emerges as the most populated at $300 \mathrm{mV}$ (and $300 \mathrm{mM} \mathrm{KCl}$ ), featuring three $\mathrm{K}^{+}$ions: at the external end of the filter, one $\mathrm{K}^{+}$is in part coordinated by the carbonyls of Y445 and in part hydrated; a second ion concurrently interacts with the carbonyls of 
G444 and V443; and the third is approximately at same level as the T442 carbonyls (Figure 3F,G). As has been noted for other channels, $\mathrm{K}^{+}$permeation proceeds through a knock-on mechanism (51-53); interestingly, we observe that this mechanism is at times mediated by water molecules permeating in between adjacent $\mathrm{K}^{+}$ions, while at other times the $\mathrm{K}^{+}$ions interact directly (Figure 3D,F, Movie S2).

The simulation calculated for the W434F mutant, which was designed identically to that carried out for Shaker-IR and is equivalent in total length, shows radically different results. We did not observe any permeation events before or after the voltage jump from 0 to $300 \mathrm{mV}$ for a total of $2.5 \mu$ s of simulation (Figure 3E, Movie S3). Instead, two $\mathrm{K}^{+}$ions persistently reside in the filter, coordinated by the carbonyls of V443 and the sidechain and backbone of T442 (Figure 3H), while multiple water molecules occupy the pocket made available near G444 and Y445 upon dilation of the filter (Figure 3J). These observations notwithstanding, we reasoned that because the selectivity filter of W434F is dilated, and not constricted, conduction ought to be feasible without a structural change provided a sufficiently strong driving force. Indeed, a second voltage jump from 300 to $450 \mathrm{mV}$ during the last $1 \mu \mathrm{s}$ of the trajectory showed 2 full permeation events (Figure 3E,I). The permeation mechanism of W434F differs from that observed for Shaker-IR, however, not only in the number of $\mathrm{K}^{+}$ions involved, but also in that $\mathrm{K}^{+}$ions do not dwell at the dilation site near G444 and Y445 before reaching the external solution (Figure 3E, Movie S3). Analogous voltage jumps from 300 to $600 \mathrm{mV}$ and from 300 to $900 \mathrm{mV}$ led to a larger number of $\mathrm{K}^{+}$ion permeation events, but nevertheless the preferred species at this distortion was again water (not shown). From this data, we can extract two tentative conclusions: first, the reconfiguration the external pore observed in the W434F mutant clearly impairs the conducting properties of the selectivity filter in comparison to Shaker-IR at the same conditions; second, this reconfiguration impairs conduction not by blocking the ion pathway but rather by imposing a mechanism of $\mathrm{K}^{+}$ permeation that entails significantly larger energetic barriers. It will be of great interest to further dissect and quantify these phenomena in future studies. 


\section{Discussion}

Our objective was to solve structures of the Shaker $\mathrm{Kv}$ channel to understand its mechanism of C-type inactivation, which is likely shared by many other eukaryotic channels. The structure of Shaker-IR provides an important framework for future studies aimed at exploring voltage-dependent gating mechanisms, and for reexamining a large body of functional studies to gain new mechanistic insights $(1,4,5)$. The value of structures of the same protein used in extensive functional studies is exemplified here with the W434F mutation, which renders the channel effectively non-conducting by greatly promoting C-type inactivation $(23,29,41)$.

The conformational rearrangement and dilation of the selectivity filter that we observe in W434F are supported by many functional studies exploring the mechanism of C-type inactivation. The hydrogen bond between D447 and W434 that breaks off in the structure of W434F has been shown to stabilize the open state (40), with mutation of either residue dramatically promoting Ctype inactivation $(29,31,40)$. T449 is a particularly critical residue for C-type inactivation because polar substitutions speed C-type inactivation and hydrophobic substitutions slow inactivation (24). Indeed, hydrophobic substitutions at T449 so dramatically slow C-type inactivation that they can rescue normal ion conduction in W434F (28). The structure of Shaker-IR reveals that T449 is partially buried as it is positioned within $4 \AA$ of the aromatic ring of Y445 in the adjacent subunit, whereas it becomes completely solvent exposed in the C-type inactive state. Hydrophobic substitution at 449 would thus be expected to stabilize the conducting conformation (Figure 2). Remarkably, the large displacement of T449 in W434F places this sidechain within 3.2 A of D447 for one of the resolved rotamers for the D447 sidechain (Figure 2), which explains why Cys substitutions at position 449 generate a strong metal binding site that forms at much faster rates when channels are inactivated (25). Although these metal bridges had been envisioned to form between $\mathrm{T} 449 \mathrm{C}$ residues in opposing subunits across the pore axis, thereby supporting the theory that C-type inactivation entails the collapse of the filter, our structures reveal an alternate interpretation, namely that the metal bridges form between T449C and D447 within the same subunit in the C-type inactivated state. The increased solvent exposure of T449 and P450 observed in the rapidly inactivating mutant is also consistent with the enhanced rate of modification of introduced Cys residues at these positions by thiol reactive compounds when Shaker C-type inactivates (27). It is also worth clarifying that our structures are not at all inconsistent with the observation that externally-applied tetraethyl ammonium or $\mathrm{K}^{+}$ions can interfere with C-type 
inactivation. While this observation had been rationalized using a foot-in-the-door analogy, in support of the notion the filter collapses upon inactivation $(25,27)$, a mechanism whereby binding of these ions within the selectivity filter hinders inactivation by precluding its dilation is equally plausible (15). All that is required to explain this particular experimental observation is for the conducting filter to interact more favorably with external ions than the inactive state; the structure of C-type inactivated Shaker is clearly consistent with this premise, as it lacks the external ionbinding sites seen in the activated state.

The structural changes we observe in the ion selectivity filter also provide important insight into how inactivation of the selectivity filter might be coupled with other conformational mechanisms elsewhere in the channel. For example, in KcsA opening of the internal pore is thought to trigger collapse of the selectivity filter because a bulky Phe in the pore-lining M2 helix interacts with a conserved Thr at the base of the ion selectivity filter (Fig. S10D) (37, 54). Our structures suggest that this coupling mechanism is unlikely to apply for the Shaker Kv channel, as the base of the ion selectivity filter is unchanged when comparing activated and C-type inactivated states (Figure 3; Fig. S10C). The reason for this difference might be that the residue equivalent to the bulky Phe in the S6 helix of Shaker is I470 (Fig. S10C), or it might simply be that the conformational changes during inactivation in KcsA are unrelated to those occurring in Shaker (Fig. S10). Indeed, C-type inactivation in Shaker is thought to be coupled instead to the conformation of the voltage-sensing domains (55-57). The current structures will greatly facilitate futures studies of this coupling mechanism.

A structural mechanism for C-type inactivation through filter dilation, when considered in the context of the groundbreaking X-ray structures of closed-state KcsA in the presence of high and low $\mathrm{K}^{+}$concentrations $(51)$, provides a new perspective on the range of functionally critical conformations required to complete the full gating cycle of a $\mathrm{Kv}$ channel. As pointed out previously (51), collapse of the filter in KcsA observed in low $\left[\mathrm{K}^{+}\right]$likely represents a conformation adopted each time the internal gate of a $\mathrm{K}^{+}$channel closes under physiological ionic conditions because in that state the filter will ultimately equilibrate with the external solution containing low $\left[\mathrm{K}^{+}\right]$(Figure 4). Collapse of the selectivity filter when the internal gate closes would assure that ion permeation is minimized in the absence of an activating stimulus (45). When the intracellular gate opens following voltage-sensor activation in a $\mathrm{Kv}$ channel, the filter will equilibrate with high $\left[\mathrm{K}^{+}\right]$inside the cell, and adopt a conducting conformation resembling that 
seen at high $\mathrm{K}^{+}$concentrations for the $\mathrm{KcsA} \mathrm{K}^{+}$channel in either closed or open states $(35,51)$, for the open-state Kv1.2/2.1 paddle chimera (7) or the open-state Shaker Kv channel reported here (Figure 4). In contrast, when Shaker and likely other eukaryotic Kv channels C-type inactivate following sustained activation, the ion selectivity filter dilates to diminish ion conduction through a mechanism distinct from the collapsed conformation adopted when the internal gate of $\mathrm{Kv}$ channels closes (Figure 4). That the ion selectivity filter adopts at least three distinct conformations during the gating cycle of a $\mathrm{Kv}$ channel beautifully illustrates how dynamic structural elements at both ends of the ion permeation pathway are employed to achieve distinct conformational states that are essential for the biological functions of $\mathrm{Kv}$ channels. It will be exciting to determine whether other $\mathrm{K}^{+}$channels use similar mechanisms to regulate ion permeation across the selectivity filter. Although structural changes related to those we see here have thus far not been resolved within the filter of K2P channels, for example, closure of those channels at the filter depletes ion occupancy in the external half of the filter while maintaining those within the inner half (58), suggesting that the mechanisms of C-type inactivation in Shaker and filter gating in $\mathrm{K} 2 \mathrm{P}$ channels may be related. 


\section{References}

1. G. Yellen, The voltage-gated potassium channels and their relatives. Nature 419, 35-42 (2002).

2. L. Y. Jan, Y. N. Jan, Voltage-gated potassium channels and the diversity of electrical signalling. J Physiol 590, 2591-2599 (2012).

3. G. Yellen, The moving parts of voltage-gated ion channels. Q Rev Biophys 31, 239-295 (1998).

4. K. J. Swartz, Sensing voltage across lipid membranes. Nature 456, 891-897 (2008).

5. F. Bezanilla, How membrane proteins sense voltage. Nat Rev Mol Cell Biol 9, 323-332 (2008).

6. A. A. Alabi, M. I. Bahamonde, H. J. Jung, J. I. Kim, K. J. Swartz, Portability of paddle motif function and pharmacology in voltage sensors. Nature 450, 370-375 (2007).

7. S. B. Long, X. Tao, E. B. Campbell, R. MacKinnon, Atomic structure of a voltagedependent K+ channel in a lipid membrane-like environment. Nature 450, 376-382 (2007).

8. D. Matthies et al., Single-particle cryo-EM structure of a voltage-activated potassium channel in lipid nanodiscs. Elife 7, (2018).

9. V. Pau, Y. Zhou, Y. Ramu, Y. Xu, Z. Lu, Crystal structure of an inactivated mutant mammalian voltage-gated K(+) channel. Nat Struct Mol Biol 24, 857-865 (2017).

10. E. Suarez-Delgado et al., KV1.2 channels inactivate through a mechanism similar to Ctype inactivation. J Gen Physiol 152, (2020).

11. T. Hoshi, W. N. Zagotta, R. W. Aldrich, Two types of inactivation in Shaker K+ channels: effects of alterations in the carboxy-terminal region. Neuron 7, 547-556 (1991).

12. J. P. Adelman, C. T. Bond, M. Pessia, J. Maylie, Episodic ataxia results from voltagedependent potassium channels with altered functions. Neuron 15, 1449-1454 (1995).

13. C. A. Hubner, T. J. Jentsch, Ion channel diseases. Hum Mol Genet 11, 2435-2445 (2002).

14. H. T. Kurata, D. Fedida, A structural interpretation of voltage-gated potassium channel inactivation. Prog Biophys Mol Biol 92, 185-208 (2006).

15. T. Hoshi, C. M. Armstrong, C-type inactivation of voltage-gated $\mathrm{K}+$ channels: pore constriction or dilation? J Gen Physiol 141, 151-160 (2013).

16. P. L. Smith, T. Baukrowitz, G. Yellen, The inward rectification mechanism of the HERG cardiac potassium channel [see comments]. Nature 379, 833-836 (1996).

17. M. T. Keating, M. C. Sanguinetti, Molecular and cellular mechanisms of cardiac arrhythmias. Cell 104, 569-580 (2001).

18. J. M. Nerbonne, R. S. Kass, Molecular physiology of cardiac repolarization. Physiol Rev 85, 1205-1253 (2005).

19. M. C. Sanguinetti, M. Tristani-Firouzi, hERG potassium channels and cardiac arrhythmia. Nature 440, 463-469 (2006).

20. J. Yan, Q. Li, R. W. Aldrich, Closed state-coupled C-type inactivation in BK channels. Proc Natl Acad Sci U S A 113, 6991-6996 (2016).

21. M. Schewe et al., A pharmacological master key mechanism that unlocks the selectivity filter gate in $\mathrm{K}(+)$ channels. Science 363, 875-880 (2019).

22. K. L. Choi, R. W. Aldrich, G. Yellen, Tetraethylammonium blockade distinguishes two inactivation mechanisms in voltage-activated K+ channels. Proc Natl Acad Sci U S A 88, 5092-5095 (1991). 
23. E. Perozo, R. MacKinnon, F. Bezanilla, E. Stefani, Gating currents from a nonconducting mutant reveal open-closed conformations in Shaker K+ channels. Neuron 11, 353-358 (1993).

24. J. Lopez-Barneo, T. Hoshi, S. H. Heinemann, R. W. Aldrich, Effects of external cations and mutations in the pore region on C-type inactivation of Shaker potassium channels. Receptors Channels 1, 61-71 (1993).

25. G. Yellen, D. Sodickson, T. Y. Chen, M. E. Jurman, An engineered cysteine in the external mouth of a $\mathrm{K}+$ channel allows inactivation to be modulated by metal binding. Biophys $J$ 66, 1068-1075 (1994).

26. T. Baukrowitz, G. Yellen, Modulation of $\mathrm{K}+$ current by frequency and external $[\mathrm{K}+]$ : a tale of two inactivation mechanisms. Neuron 15, 951-960 (1995).

27. Y. Liu, M. E. Jurman, G. Yellen, Dynamic rearrangement of the outer mouth of a K+ channel during gating. Neuron 16, 859-867 (1996).

28. Y. Yang, Y. Yan, F. J. Sigworth, The Shaker mutation T449V rescues ionic currents of W434F K ${ }^{+}$channels. Biophys J 82, 234e (2002).

29. Y. Yang, Y. Yan, F. J. Sigworth, How does the W434F mutation block current in Shaker potassium channels? J Gen Physiol 109, 779-789 (1997).

30. J. G. Starkus, L. Kuschel, M. D. Rayner, S. H. Heinemann, Ion conduction through C-type inactivated Shaker channels. J Gen Physiol 110, 539-550 (1997).

31. A. Molina, A. G. Castellano, J. Lopez-Barneo, Pore mutations in Shaker K+ channels distinguish between the sites of tetraethylammonium blockade and C-type inactivation. $J$ Physiol 499 ( Pt 2), 361-367 (1997).

32. E. M. Ogielska, R. W. Aldrich, A mutation in S6 of Shaker potassium channels decreases the $\mathrm{K}+$ affinity of an ion binding site revealing ion-ion interactions in the pore. $J$ Gen Physiol 112, 243-257 (1998).

33. E. M. Ogielska, R. W. Aldrich, Functional Consequences of a Decreased Potassium Affinity in a Potassium Channel Pore. Ion interactions and c-type inactivation. $J$ Gen Physiol 113, 347-358 (1999).

34. C. M. Armstrong, T. Hoshi, $\mathrm{K}(+)$ channel gating: C-type inactivation is enhanced by calcium or lanthanum outside. J Gen Physiol 144, 221-230 (2014).

35. L. G. Cuello, D. M. Cortes, E. Perozo, The gating cycle of a $\mathrm{K}(+)$ channel at atomic resolution. Elife 6, (2017).

36. L. G. Cuello, V. Jogini, D. M. Cortes, E. Perozo, Structural mechanism of C-type inactivation in $\mathrm{K}(+)$ channels. Nature 466, 203-208 (2010).

37. L. G. Cuello et al., Structural basis for the coupling between activation and inactivation gates in $\mathrm{K}(+)$ channels. Nature 466, 272-275 (2010).

38. J. F. Cordero-Morales, V. Jogini, S. Chakrapani, E. Perozo, A multipoint hydrogen-bond network underlying KcsA C-type inactivation. Biophys J 100, 2387-2393 (2011).

39. J. Li et al., Computational study of non-conductive selectivity filter conformations and Ctype inactivation in a voltage-dependent potassium channel. J Gen Physiol 153, (2021).

40. S. A. Pless, J. D. Galpin, A. P. Niciforovic, H. T. Kurata, C. A. Ahern, Hydrogen bonds as molecular timers for slow inactivation in voltage-gated potassium channels. Elife 2, e01289 (2013).

41. F. Bezanilla, Gating currents. J Gen Physiol 150, 911-932 (2018).

42. W. N. Zagotta, T. Hoshi, R. W. Aldrich, Restoration of inactivation in mutants of Shaker potassium channels by a peptide derived from ShB. Science 250, 568-571 (1990). 
43. T. Hoshi, W. N. Zagotta, R. W. Aldrich, Biophysical and molecular mechanisms of Shaker potassium channel inactivation. Science 250, 533-538 (1990).

44. S. D. Demo, G. Yellen, The inactivation gate of the Shaker K+ channel behaves like an open- channel blocker. Neuron 7, 743-753 (1991).

45. G. J. Soler-Llavina, M. Holmgren, K. J. Swartz, Defining the conductance of the closed state in a voltage-gated $\mathrm{K}^{+}$channel. Neuron 38, 61-67 (2003).

46. G. J. Soler-Llavina, T. H. Chang, K. J. Swartz, Functional interactions at the interface between voltage-sensing and pore domains in the Shaker $\mathrm{K}(\mathrm{v})$ channel. Neuron 52, 623634 (2006).

47. X. Tao, A. Lee, W. Limapichat, D. A. Dougherty, R. MacKinnon, A gating charge transfer center in voltage sensors. Science 328, 67-73 (2010).

48. S. K. Aggarwal, R. MacKinnon, Contribution of the S4 segment to gating charge in the Shaker K+ channel. Neuron 16, 1169-1177 (1996).

49. S. A. Seoh, D. Sigg, D. M. Papazian, F. Bezanilla, Voltage-sensing residues in the S2 and S4 segments of the Shaker K+ channel. Neuron 16, 1159-1167 (1996).

50. Y. Liu, M. Holmgren, M. E. Jurman, G. Yellen, Gated access to the pore of a voltagedependent K+ channel. Neuron 19, 175-184 (1997).

51. Y. Zhou, J. H. Morais-Cabral, A. Kaufman, R. MacKinnon, Chemistry of ion coordination and hydration revealed by a $\mathrm{K}+$ channel- Fab complex at 2.0 A resolution. Nature 414, 4348. (2001).

52. J. H. Morais-Cabral, Y. Zhou, R. MacKinnon, Energetic optimization of ion conduction rate by the K+ selectivity filter. Nature 414, 37-42. (2001).

53. A. Mironenko, U. Zachariae, B. L. de Groot, W. Kopec, The Persistent Question of Potassium Channel Permeation Mechanisms. J Mol Biol 433, 167002 (2021).

54. A. J. Labro, D. M. Cortes, C. Tilegenova, L. G. Cuello, Inverted allosteric coupling between activation and inactivation gates in $\mathrm{K}(+)$ channels. Proc Natl Acad Sci U S A 115, 5426-5431 (2018).

55. R. Olcese, R. Latorre, L. Toro, F. Bezanilla, E. Stefani, Correlation between charge movement and ionic current during slow inactivation in Shaker $\mathrm{K}+$ channels. J Gen Physiol 110, 579-589 (1997).

56. E. Loots, E. Y. Isacoff, Protein rearrangements underlying slow inactivation of the Shaker K+ channel. J Gen Physiol 112, 377-389 (1998).

57. H. P. Larsson, F. Elinder, A conserved glutamate is important for slow inactivation in $\mathrm{K}+$ channels. Neuron 27, 573-583 (2000).

58. M. Lolicato et al., K2P channel C-type gating involves asymmetric selectivity filter orderdisorder transitions. Sci Adv 6, (2020).

59. M. S. Rana, X. Wang, A. Banerjee, An Improved Strategy for Fluorescent Tagging of Membrane Proteins for Overexpression and Purification in Mammalian Cells. Biochemistry 57, 6741-6751 (2018).

60. A. Goehring et al., Screening and large-scale expression of membrane proteins in mammalian cells for structural studies. Nature protocols 9, 2574-2585 (2014).

61. D. N. Mastronarde, Automated electron microscope tomography using robust prediction of specimen movements. J Struct Biol 152, 36-51 (2005).

62. J. Zivanov et al., New tools for automated high-resolution cryo-EM structure determination in RELION-3. Elife 7, (2018). 
63. A. Punjani, J. L. Rubinstein, D. J. Fleet, M. A. Brubaker, cryoSPARC: algorithms for rapid unsupervised cryo-EM structure determination. Nature methods 14, 290-296 (2017).

64. S. Q. Zheng et al., MotionCor2: anisotropic correction of beam-induced motion for improved cryo-electron microscopy. Nature methods 14, 331-332 (2017).

65. A. Rohou, N. Grigorieff, CTFFIND4: Fast and accurate defocus estimation from electron micrographs. J Struct Biol 192, 216-221 (2015).

66. E. F. Pettersen et al., UCSF Chimera--a visualization system for exploratory research and analysis. J Comput Chem 25, 1605-1612 (2004).

67. P. Emsley, B. Lohkamp, W. G. Scott, K. Cowtan, Features and development of Coot. Acta Crystallogr D Biol Crystallogr 66, 486-501 (2010).

68. P. D. Adams et al., PHENIX: a comprehensive Python-based system for macromolecular structure solution. Acta Crystallogr D Biol Crystallogr 66, 213-221 (2010).

69. S. B. Pandit, J. Skolnick, Fr-TM-align: a new protein structural alignment method based on fragment alignments and the TM-score. BMC bioinformatics 9, 531 (2008).

70. O. S. Smart, J. G. Neduvelil, X. Wang, B. A. Wallace, M. S. Sansom, HOLE: a program for the analysis of the pore dimensions of ion channel structural models. J Mol Graph 14, 354-360, 376 (1996).

71. L. Zhang, J. Hermans, Hydrophilicity of cavities in proteins. Proteins 24, 433-438 (1996).

72. B. R. Brooks et al., CHARMM: The Biomolecular Simulation Program. Journal of Computational Chemistry 30, 1545-1614 (2009).

73. J. Huang et al., CHARMM36m: an improved force field for folded and intrinsically disordered proteins. Nature methods 14, 71-73 (2017).

74. R. B. Best et al., Optimization of the additive CHARMM all-atom protein force field targeting improved sampling of the backbone phi, psi and side-chain chi(1) and chi(2) dihedral angles. J Chem Theory Comput 8, 3257-3273 (2012).

75. J. B. Klauda et al., Update of the CHARMM all-atom additive force field for lipids: validation on six lipid types. J Phys Chem B 114, 7830-7843 (2010).

76. T. A. Wassenaar, H. I. Ingolfsson, R. A. Bockmann, D. P. Tieleman, S. J. Marrink, Computational Lipidomics with insane: A Versatile Tool for Generating Custom Membranes for Molecular Simulations. J Chem Theory Comput 11, 2144-2155 (2015).

77. S. Pronk et al., GROMACS 4.5: a high-throughput and highly parallel open source molecular simulation toolkit. Bioinformatics 29, 845-854 (2013).

78. D. H. de Jong et al., Improved Parameters for the Martini Coarse-Grained Protein Force Field. J Chem Theory Comput 9, 687-697 (2013).

79. S. J. Marrink, A. H. de Vries, A. E. Mark, Coarse grained model for semiquantitative lipid simulations. Journal of Physical Chemistry B 108, 750-760 (2004).

80. L. Monticelli et al., The MARTINI Coarse-Grained Force Field: Extension to Proteins. $J$ Chem Theory Comput 4, 819-834 (2008).

81. S. J. Marrink, H. J. Risselada, S. Yefimov, D. P. Tieleman, A. H. de Vries, The MARTINI force field: coarse grained model for biomolecular simulations. J Phys Chem B 111, 78127824 (2007).

82. T. A. Wassenaar, K. Pluhackova, R. A. Bockmann, S. J. Marrink, D. P. Tieleman, Going Backward: A Flexible Geometric Approach to Reverse Transformation from Coarse Grained to Atomistic Models. J Chem Theory Comput 10, 676-690 (2014).

83. J. C. Phillips et al., Scalable molecular dynamics on CPU and GPU architectures with NAMD. The Journal of chemical physics 153, 044130 (2020). 
84. G. Fiorin, M. L. Klein, J. Henin, Using collective variables to drive molecular dynamics simulations. Molecular Physics 111, 3345-3362 (2013).

85. D. E. Shaw et al., paper presented at the SC14: International Conference for High Performance Computing, Networking, Storage and Analysis, 2014.

86. S. Nosé, A Molecular Dynamics Method for Simulations in the Canonical Ensemble. Molecular Physics 52, 255-268 (1984).

87. W. G. Hoover, Canonical Dynamics - Equilibrium Phase-Space Distributions. Physical Review A 31, 1695-1697 (1985).

88. G. J. Martyna, D. J. Tobias, M. L. Klein, Constant-Pressure Molecular-Dynamics Algorithms. Journal of Chemical Physics 101, 4177-4189 (1994).

89. Y. Shan, J. L. Klepeis, M. P. Eastwood, R. O. Dror, D. E. Shaw, Gaussian split Ewald: A fast Ewald mesh method for molecular simulation. The Journal of chemical physics 122, 54101 (2005).

90. A. C. Pan et al., Atomic-level characterization of protein-protein association. P Natl Acad Sci USA 116, 4244-4249 (2019).

91. M. O. Jensen et al., Mechanism of Voltage Gating in Potassium Channels. Science 336, 229-233 (2012). 
Acknowledgments: We thank Andres Jara-Oseguera, Miguel Holmgren, Mark Mayer and members of the Swartz laboratory for helpful discussion, and Huaibin Wang in the NIH MultiInstitute Cryo-EM Facility (MICEF) for assistance in acquiring cryo-EM data. This work utilized the NIH MICEF and computational resources of the NIH HPC Biowulf cluster (http://hpc.nih.gov)

\section{Funding:}

This research was supported by the Intramural Research Programs of the National Institute of Neurological Disorders and Stroke, NIH, Bethesda, MD to KJS, and the National Heart Blood and Lung Institute, NIH, Bethesda, MD to J.J. and J.F.G.

\section{Author contributions:}

Conceptualization: $\mathrm{CB}, \mathrm{XT}$, KJS

Methodology: XT, CB, RS, AIF, KH, TC, JJ, JDF, KJS

Investigation: XT, CB, RS, AIF, TC, JDF

Visualization: XT, CB, RS, AIF, KH, JDF, KJS

Funding acquisition: JJ, JDF, KJS

Project administration: CB, JDF, KJS

Supervision: JDF, KJS

Writing - original draft: XT, CB, RS, AIF, KH, TC, JJ, JDF, KJS

Writing - review \& editing: XT, CB, RS, AIF, KH, TC, JJ, JDF, KJS

Competing interests: Authors declare that they have no competing interests.

Data and materials availability: The map of wild-type Shaker-IR and the W434F mutant have been deposited in the Electron Microscopy Data Bank (EMDB) under accession codes EMDXXXXX and EMD-XXXXX, respectively. Models of wild-type Shaker-IR and the W434F mutant have been deposited in the Protein Data Bank with accession codes XXXX and XXXX, respectively.

\section{Supplementary Materials}

Materials and Methods

Figs. S1 to S10

Tables S1 to S2

Captions for Movies S1 to S3

Movies S1 to S3 
A

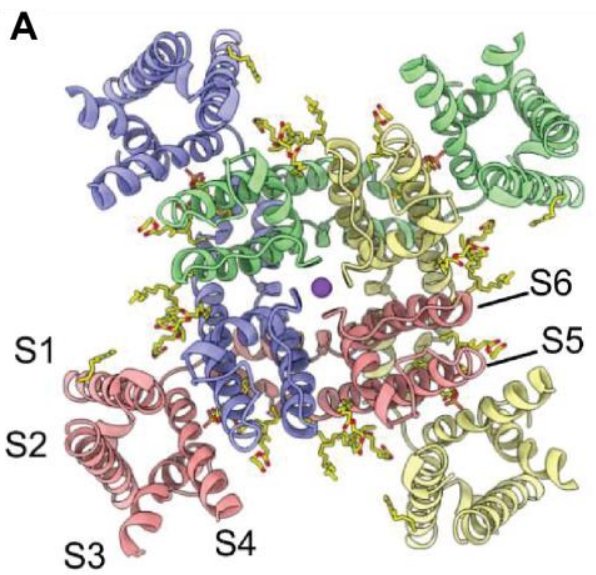

C
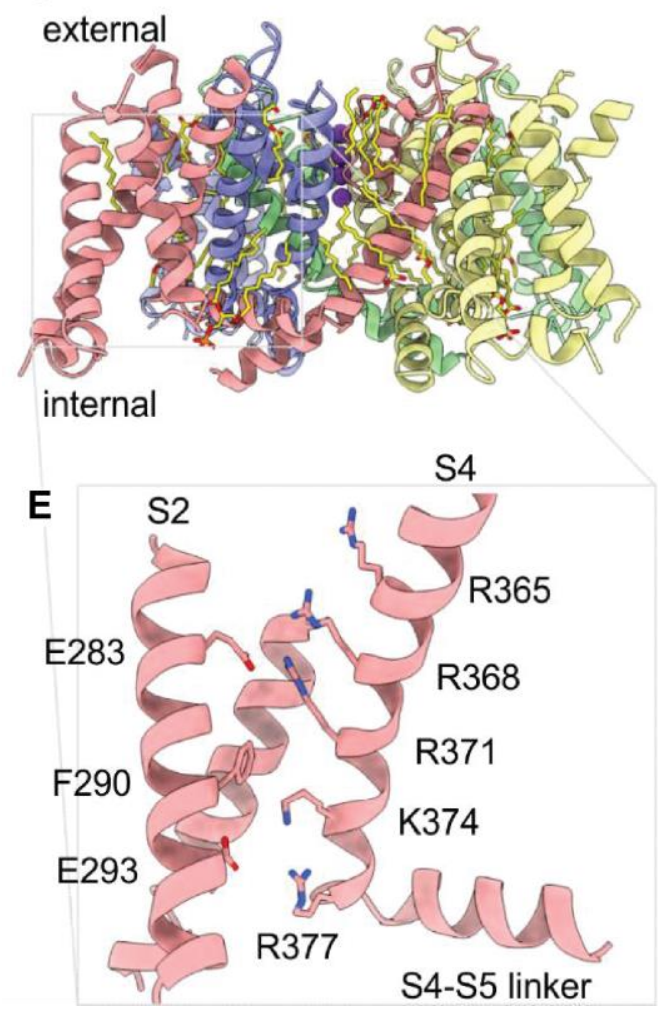

B

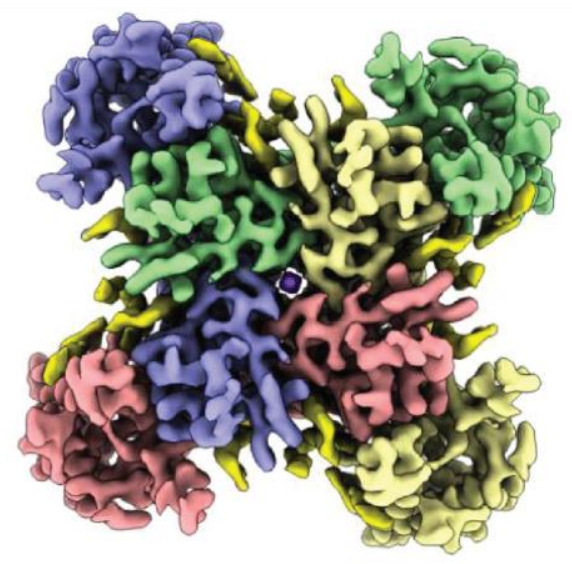

D

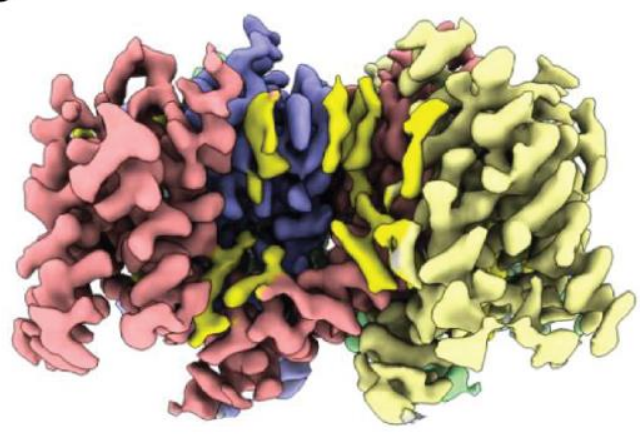

F

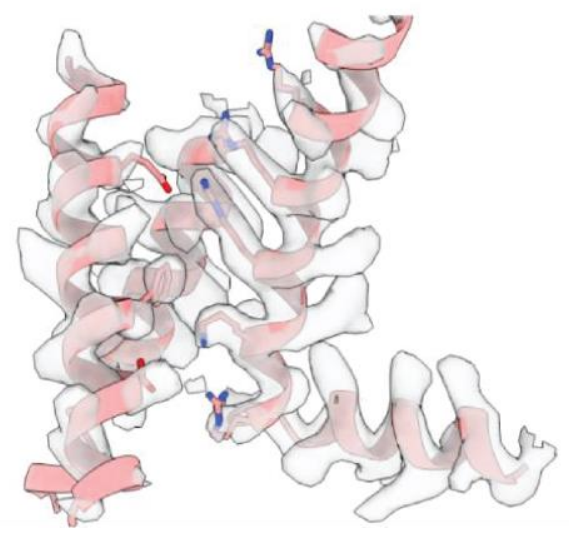

Figure 1| Structure of the Shaker-IR Kv channel in lipid nanodisc

A and B, View of Shaker Kv channel model (A) and EM map (B) from an extracellular perspective.

C and D, Transmembrane side-view of Shaker-IR Kv channel model (C) and EM map (D). Each subunit is shown in different colors and EM densities that appear to be lipid molecules are colored in bright yellow. $\mathbf{E}$ and $\mathbf{F}$, Close-up view of a voltage-sensing domain. The voltage-sensing domain highlighted with a gray box in $\mathrm{c}$ is shown without (E) and with (F) EM density. 
bioRxiv preprint doi: https://doi.org/10.1101/2021.09.21.461258; this version posted September 21, 2021. The copyright holder for this preprint (which was not certified by peer review) is the author/funder. This article is a US Government work. It is not subject to copyright under 17 USC 105 and is also made available for use under a CCO license.
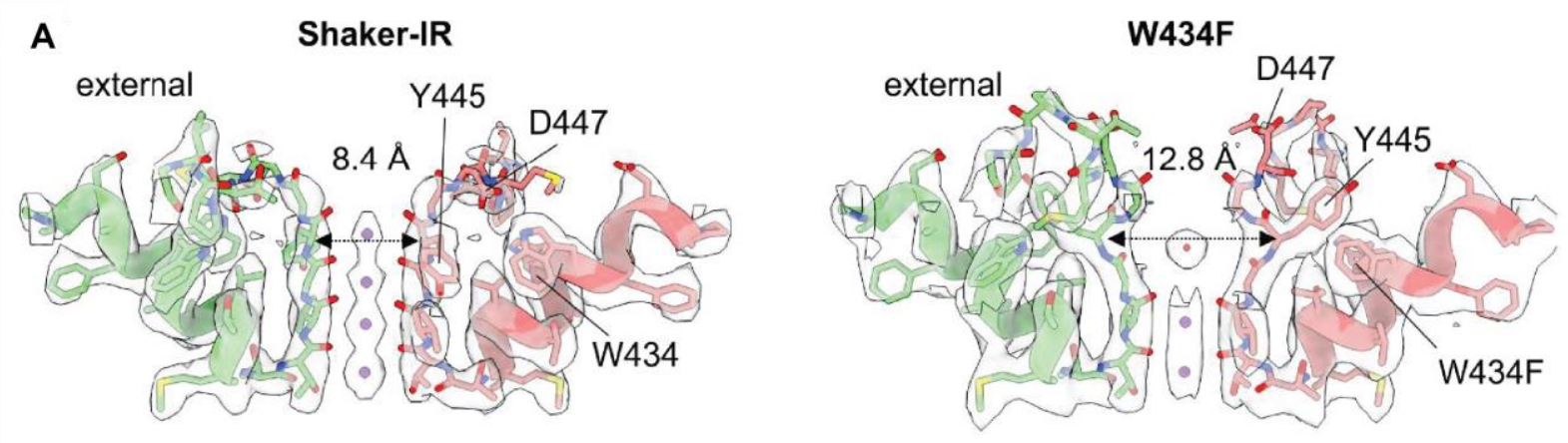

B
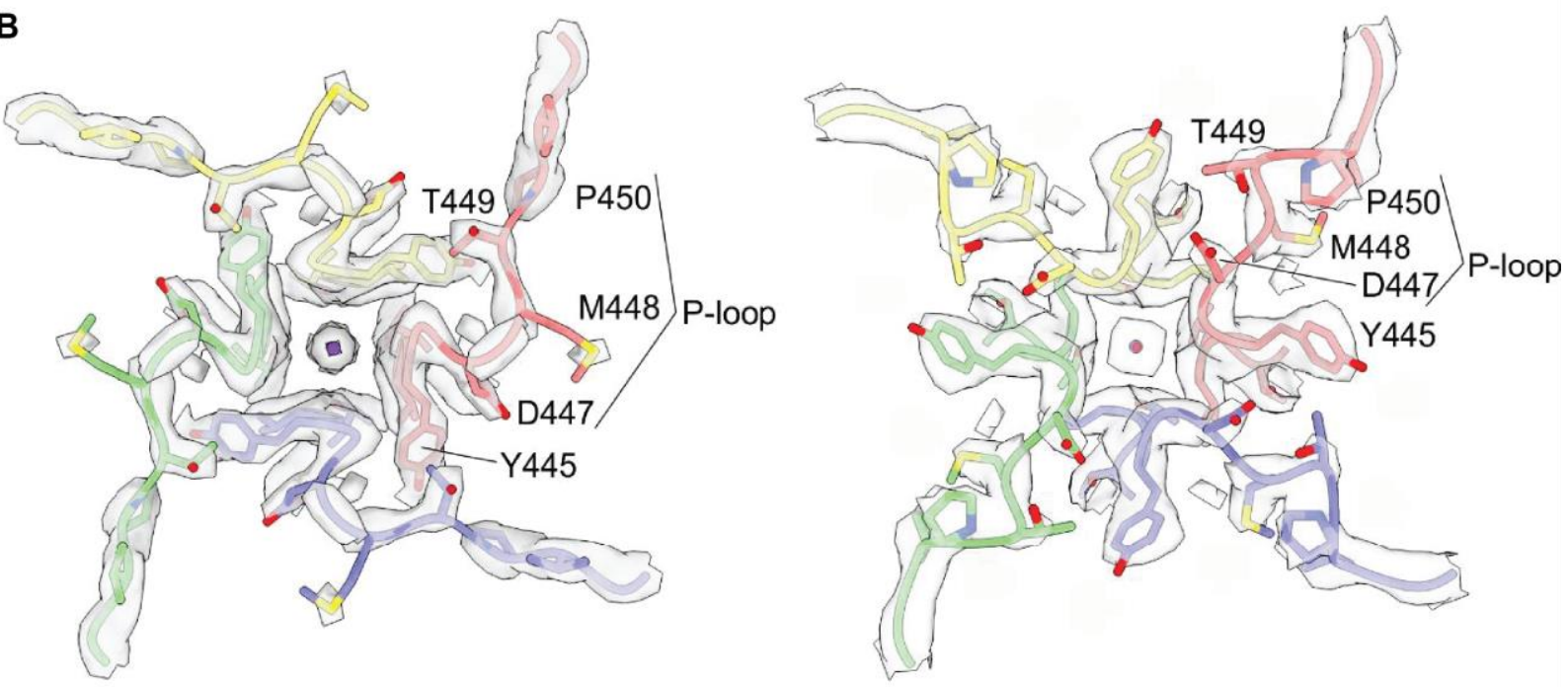

C

D Shaker-IR
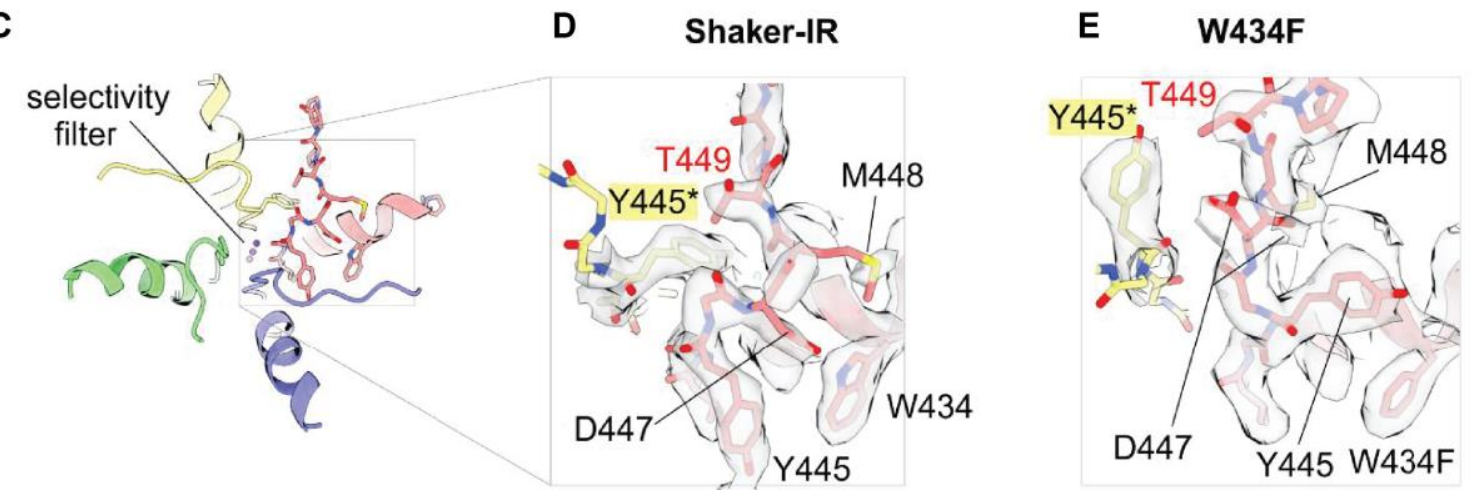


\section{$\leftarrow$ Figure 2| Structure of the external pore of wild-type Shaker-IR and the W434F mutant}

A, Side-view of the selectivity filter of Shaker-IR (left) and W434F (right). The distance between Y445 CA is shown to highlight the dilation of the selectivity filter in W434F. B, View of the pore domain of Shaker-IR (left) and W434F (right) viewed from an extracellular perspective. Residues experiencing a large movement are labeled to highlight structural reorientation of the outer pore domain in W434F. C, Outer-pore region of the Shaker-IR viewed from a tilted extracellular perspective. The grey box is zoomed in to highlight detailed structural changes of side chains in $\mathbf{D}$ for Shaker-IR and $\mathbf{E}$ for W434F. Residues experiencing a large conformational change are shown and labeled. 
bioRxiv preprint doi: $h$ ttps://doi.org/10.1101/2021.09.21.461258; this version posted September 21,2021 . The copyright holder for this preprint (which was not certified by peer review) is the author/funder. This article is a US Government work. It is not subject to copyright under 17 USC 105 and is also made available for use under a CCO license.
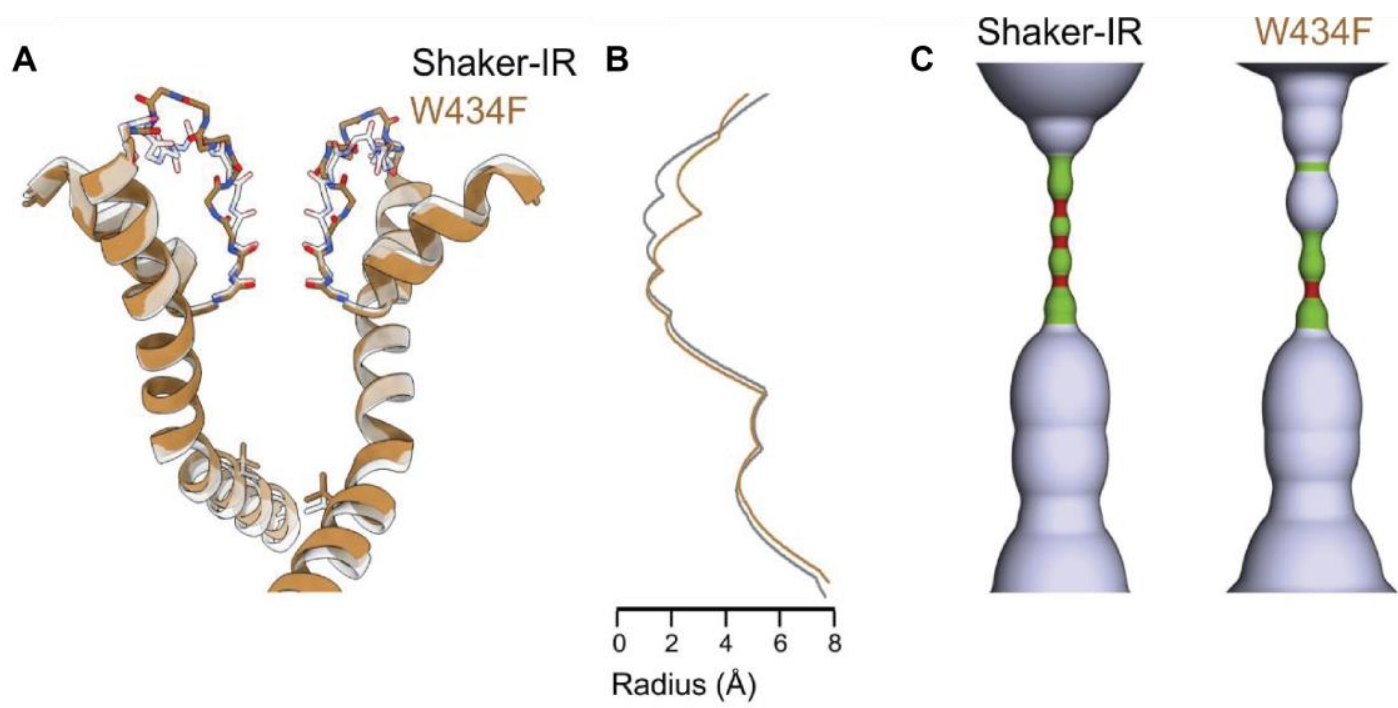

Radius $(\AA)$
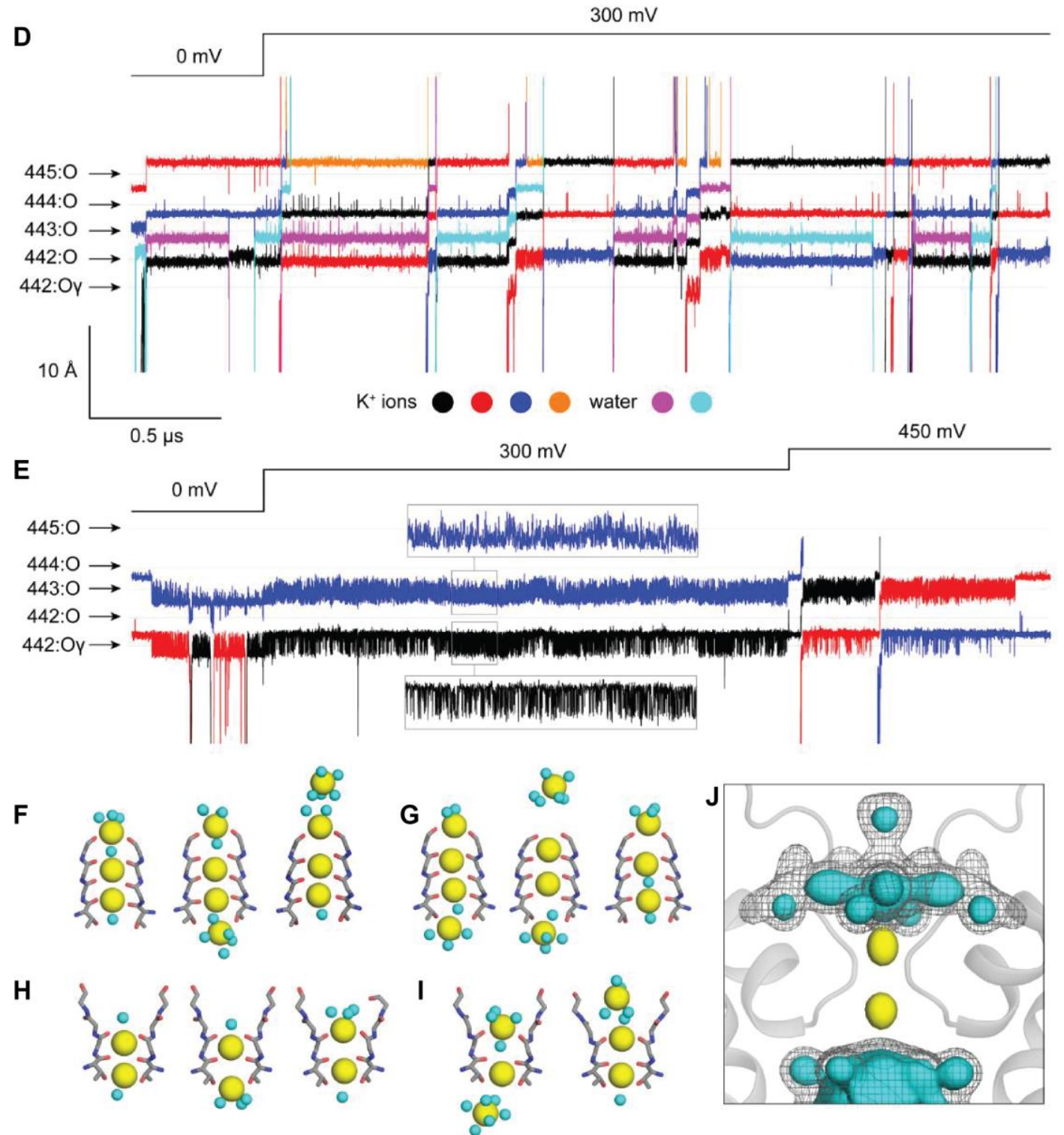


\section{$\leftarrow$ Figure 3| Conformational changes and altered ion interactions with the filter during}

\section{C-type inactivation in Shaker Kv channels}

A, Superposition of pore-lining regions for Shaker-IR and the W434F mutant. V478 is shown to mark the position of the internal S6 gate that prevents ion permeation across the internal pore when the Shaker Kv channel closes (50). B, Plot of pore radius for Shaker-IR and the W434F mutant. C, Hole diagrams illustrating the ion permeation pathways for Shaker-IR and W434F. Radii $\leq 1$ $\AA$ are shown in red, radii $>1 \AA$ and $\leq 2 \AA$ are shown in green, and radii larger than $2 \AA$ are shown in light blue. The plot in b and the diagram in $\mathrm{c}$ are aligned with the protein model in a. D, Molecular dynamics simulation of $\mathrm{K}^{+}$permeation across the pore domain of Shaker-IR. The figure shows time-series of the position of $\mathrm{K}^{+}$ions as they traverse or transiently enter the selectivity filter; time-series of water molecules traversing the filter are also shown. The time resolution is 120 ps. The relative position of coordinating backbone/sidechain oxygens in residues 442-445 is indicated. Note no voltage was applied during the first $0.5 \mu \mathrm{s}$ of the trajectory; a jump to $300 \mathrm{mV}$ was introduced at this point and sustained for $3 \mu$ s thereafter. E, Same as c, for W434F mutant. Note that in this case a second voltage jump from 300 to $450 \mathrm{mV}$ was introduced at $2.5 \mu \mathrm{s}$ and sustained for $1 \mu$ s thereafter. F,G, Snapshots of two $\mathrm{K}^{+}$permeation events during the simulation of Shaker-IR. $\mathrm{K}^{+}$ions and water molecules are shown as yellow and cyan spheres, respectively. The elapsed times between snapshots are approximately 3 and 4 ns, respectively. H, Representative configurations of the selectivity filter in the simulation of the W434F mutant at 300 $\mathrm{mV}$. Both ions fluctuate but fail to permeate in $2 \mu \mathrm{s}$ of trajectory under this condition. I, Snapshots of a $\mathrm{K}^{+}$permeation event during the simulation of $\mathrm{W} 434 \mathrm{~F}$, after the jump to $450 \mathrm{mV}$. The elapsed time between snapshots is approximately $8 \mathrm{~ns}$. $\mathbf{J}$, Calculated iso-density maps for $\mathrm{K}^{+}$(yellow surface) and water (cyan surface, gray mesh) near and within the selectivity filter of W434F, based on analysis of $2 \mu \mathrm{s}$ of trajectory under $300 \mathrm{mV}$. For water, two iso-values are used, to illustrate the emergence of well-defined, 4-fold symmetric structures resulting from interactions with ions and protein as well as confinement. 


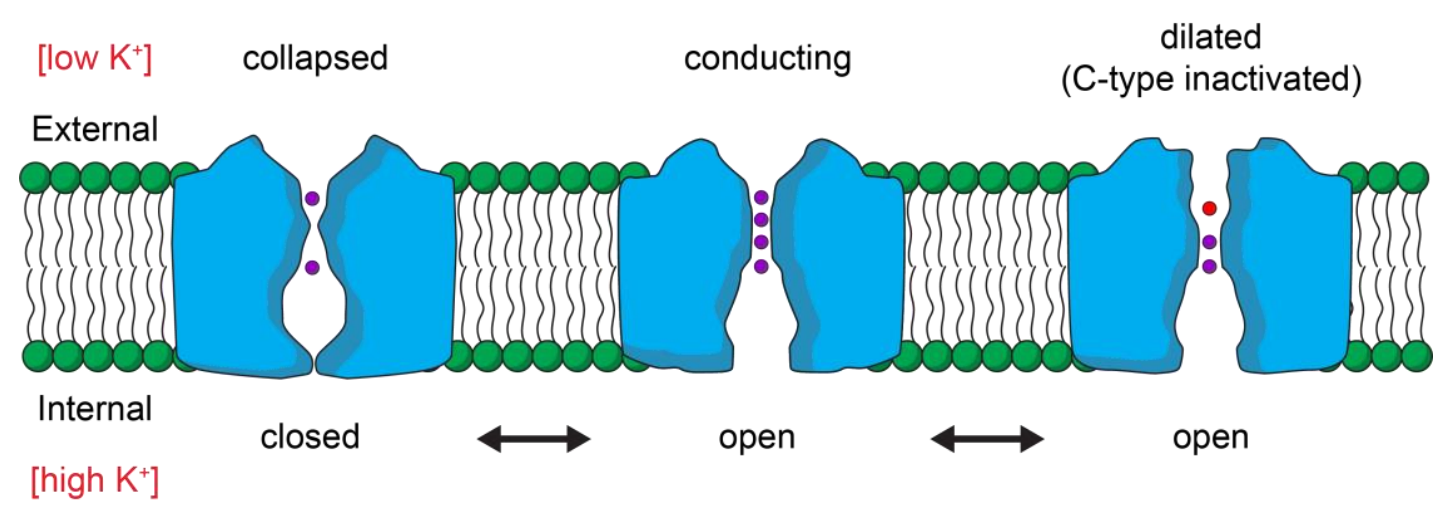

\section{Figure 4| Gating cycle of the pore domain of a Kv channel}

Cartoons illustrating dynamic changes in structure of the ion conduction pore during the gating cycle of a $\mathrm{Kv}$ channel. When the voltage-sensing domains are resting at negative membrane voltages and the internal S6 gate is closed, the filter would equilibrate with the external solution containing low potassium and collapse. With voltage-sensor activation and opening of the internal S6 gate, the filter will equilibrate with high potassium inside the cell and adopt a conducting conformation. With prolonged activation, the filter C-type inactivates and collapses to diminish ion permeation. Four $\mathrm{K}^{+}$ion binding sites (purple) within the filter can be occupied in the conducting state, whereas in the C-type inactivated state ions primarily occupy only the internal half of the filter and water molecules (red) likely occupies the dilated external part of the filter. 


\section{Supplementary Materials for}

Structure of the Shaker Kv channel and mechanism of slow C-type inactivation

Xiao-Feng Tan, Chanhyung Bae, Robyn Stix, Anabel I. Fernandez, Kate Huffer,Tsg-Hui

Chang, Jiansen Jiang, José D. Faraldo-Gómez and Kenton J. Swartz*

Correspondence to: swartzk@ninds.nih.gov

\section{This PDF file includes:}

Materials and Methods

Figs. S1 to S10

Tables $\mathrm{S} 1$ to $\mathrm{S} 2$

Captions for Movies S1 to S3

Other Supplementary Materials for this manuscript include the following:

Movies S1 to S3 


\section{Materials and Methods}

\section{Shaker Kv channel expression using Baculovirus and mammalian expression system}

To produce the Shaker Kv channel for cryoEM, WT and W434F channels were cloned into the pEG vector in which EGFP was substituted with mVenus (62) and expressed in tsA201 cells using the previously published Baculovirus-mammalian expression system with a few minor modifications (63) . Briefly, P1 virus was generated by transfecting Sf9 cells ( 2.5 million cells on a T25 flask with a vent cap) with 50 - 100 ng of fresh Bacmid using Cellfectin. After $4-5$ days incubation in a humidified $28{ }^{\circ} \mathrm{C}$ incubator, the cell culture media was collected by centrifugation (3,000g x $10 \mathrm{~min})$, supplemented with $2 \% \mathrm{FBS}$, and filtered through a $0.45 \mu \mathrm{m}$ filter to harvest the $\mathrm{P} 1$ virus. To amplify the $\mathrm{P} 1$ virus, $\sim 500 \mathrm{ml} \mathrm{Sf9}$ cell cultures at a $\sim 1.5$ million cells $/ \mathrm{ml}$ density were infected with $1-200 \mu \mathrm{l}$ of the virus and incubated in a $28{ }^{\circ} \mathrm{C}$ shaking incubator for 3 days. The cell culture media was then collected by centrifugation $(5,000 \mathrm{~g}$ x $20 \mathrm{~min})$, supplemented with $2 \%$ FBS, and filter through $0.45 \mu \mathrm{m}$ filter to harvest $\mathrm{P} 2$ virus. The volume of P1 virus used for the amplification was determined by carrying out a small-scale amplification screening in which 10 $\mathrm{ml} \mathrm{Sf9}$ cell cultures at the same density were infected with different volume of P1 virus and harvested after 3 days to transduce tsA201 cells and compare the expression level of Shaker Kv channels using mVenus fluorescence intensity. The P2 virus was protected from light using aluminum foil and stored at $4{ }^{\circ} \mathrm{C}$ until use. To express the Shaker channels, tsA201 cells at $\sim 1.5$ million cells/ml in Freestyle medium with $2 \%$ FBS were transduced with $10 \%$ (v/v) P2 virus and incubated at a $37{ }^{\circ} \mathrm{C} \mathrm{CO}_{2}$ incubator. To boost the protein expression, sodium butyrate (2 $\mathrm{M}$ stock in $\mathrm{H}_{2} \mathrm{O}$ ) was added to $10 \mathrm{mM}$ at $\sim 16$ hours of post-transduction. The culture was continued at the $37{ }^{\circ} \mathrm{C} \mathrm{CO}_{2}$ incubator for another 24 hours, and the cells were harvested by centrifugation $(5,000 \mathrm{~g}$ $\mathrm{x} 20 \mathrm{~min}$ ) and frozen at $-80^{\circ} \mathrm{C}$ until use.

\section{Shaker Kv channel purification}

Prior to extraction of the Shaker channels from tsA201 cells, membrane fractionation was carried out using a hypotonic solution and ultracentrifugation. The cells were first resuspended in a hypotonic solution $(20 \mathrm{mM}$ Tris $\mathrm{pH} 7.5$ and $10 \mathrm{mM} \mathrm{KCl}$ ) with protease inhibitors (pepstatin, aprotinin, leupeptin, benzamidine, trypsin inhibitor, PMFS) using a Dounce homogenizer, incubated at $4{ }^{\circ} \mathrm{C}$ for $\sim 30$ minutes, and centrifuged at $1,000 \mathrm{~g}$ for 10 minutes to remove cell debris. 
The supernatant was ultracentrifuged for 1 hour (45,000 rpm, Beckman Ti45 rotor) and collected membranes were stored at $-80{ }^{\circ} \mathrm{C}$ until use. To purify Shaker $\mathrm{Kv}$ channels, the fractionated membranes were resuspended in an extraction buffer $(50 \mathrm{mM}$ Tris $\mathrm{pH} 7.5,150 \mathrm{mM} \mathrm{KCl}, 2 \mathrm{mM}$ TCEP, $50 \mathrm{mM}$ DDM, $5 \mathrm{mM}$ CHS with the protease inhibitor mixture used above) and extracted for 1 hour at $4{ }^{\circ} \mathrm{C}$. The solution was clarified by centrifugation $(12,000 \mathrm{~g}$ x $10 \mathrm{~min})$ and incubated with CoTALON resins at $4{ }^{\circ} \mathrm{C}$ for 1 hour, at which point the mixture was transferred to an empty disposable column (Econo-Pac $®$ Biorad). The resin was washed with 10 column volume of Buffer A (50 mM Tris pH 7.5, $150 \mathrm{mM} \mathrm{KCl,} 1 \mathrm{mM}$ DDM, $0.1 \mathrm{mM} \mathrm{CHS}$, and $0.1 \mathrm{mg} / \mathrm{ml}$ porcine brain total lipid extract) with $10 \mathrm{mM}$ imidazole, and bound proteins were eluted with Buffer A with 250 $\mathrm{mM}$ imidazole. The eluate was concentrated using Amicon Ultra (100kDa) to $\sim 350-450 \mu 1$ and loaded onto a Superose6 (10x300mm) gel filtration column and separated with Buffer A. All purification steps described above was carried out at $4{ }^{\circ} \mathrm{C}$ or on ice.

\section{Lipid nanodisc reconstitution of the Shaker Kv channel}

Lipid nanodisc reconstitution was performed following the previously published methods with minor modifications (20). On the day of nanodisc reconstitution, the Shaker $\mathrm{Kv}$ channel purified by Superose6 in detergent was concentrated to $\sim 1-3 \mathrm{mg} / \mathrm{ml}$ and incubated with histidine tagged MSP1E3D1 and 3:1:1 mixture of 1-palmitoyl-2-oleoyl-sn-glycero-3-phosphocholine (POPC), 1palmitoyl-2-oleoyl-sn-glycero-3-phospho-(1'-rac-glycerol) (POPG) and 1-palmitoyl-2-oleoyl-snglycero-3-phosphoethanolamine (POPE) for 30 minutes at room temperature. The mixture was transferred to a tube with Biobeads ( 30-50 fold of detergent; w/w) and incubated at room temperature for $\sim 3$ hours in the presence of TEV protease (prepared in-house) and $2 \mathrm{mM}$ TCEP to remove $\mathrm{N}$-terminal fusion protein including poly-histidine and mVenus tag. The reconstituted protein was loaded onto Superose6 column (10x300mm) and separated using $20 \mathrm{mM}$ Tris and 150 $\mathrm{mM} \mathrm{KCl}$ buffer at $4{ }^{\circ} \mathrm{C}$. The success of nanodisc reconstitution was confirmed by collecting separated fractions and running SDS-PAGE to verify the presence of Shaker Kv and MSP1E3D1 bands at a similar ratio. Typically, optimal reconstitution required the incubation of 1:10:200 or 1:10:400 molar ratio of tetrameric Shaker Kv, MSP1E3D1, and the lipid mixture. 


\section{Cryo-EM sample preparation and data acquisition}

Concentrated samples of Shaker-IR or the W434F mutant in nanodiscs (3 $\mu \mathrm{L})$ were applied to glow-discharged Quantifoil grids (R 1.2/1.3 Cu 300 mesh). The grids were blotted for $2.5 \mathrm{~s}$, blotforce 4, $100 \%$ humidity, at $16^{\circ} \mathrm{C}$ using a FEI Vitrobot Mark IV (Thermo Fisher), followed by plunging into liquid ethane cooled by liquid nitrogen. Images were acquired using an FEI Titan Krios equipped with a Gatan LS image energy Filter (slit width $20 \mathrm{eV}$ ) operating at $300 \mathrm{kV}$. A Gatan K3 Summit direct electron detector was used to record movies in super-resolution mode with a nominal magnification of $105,000 x$, resulting in a calibrated pixel size of $0.43 \AA$ per pixel. The typical defocus values ranged from -0.5 to $-1.5 \mathrm{um}$. Exposures of $1.6 \mathrm{~s}$ were dose-fractionated into 32 frames, resulting in a total dose of 52 e- $\AA-2$. Images were recorded using the automated acquisition program SerialEM (64).

\section{Image processing}

All processing was completed in RELION (65) and cryoSPARC (66). The beam-induced image motion between frames of each dose-fractionated micrograph was corrected and binned by 2 using MotionCor2 (67) and contrast transfer function (CTF) estimation was performed using CTFFIND4 (68). Micrographs were selected and those with outliers in defocus value and astigmatism, as well as low resolution (>5 $\AA$ ) reported by CTFFIND4 were removed. The initial set of particles from 300 micrographs were picked using Gautomatch (https://www2.mrc-

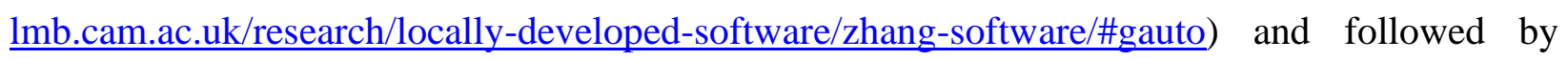
reference-free 2D Classification in RELION. The good classes were then used as template to pick particles from all selected micrographs using Gautomatch. 4,934,881 (Shaker-IR) and 7,446,104 (W434F) particles were picked and extracted with 2x downscaling (pixel size $1.72 \AA$ ). Several rounds of reference-free 2D Classification were performed to remove ice spot, contaminants and bad particles, yielding 2,246,076 (WT) and 2,347,164 (W434F) particles, respectively. The good particles were 3D classified using reference generated by 3D initial model in C1 symmetry. Good classes were then selected and followed by further 2D Classification and 3D Classification. The classes which show good features within the transmembrane domains were merged. After removing duplicate particles, the selected particles were re-extracted using box size 296 pixels 
without binning (pixel size $0.86 \AA$ ). To get good reconstruction within the transmembrane domain, the re-extracted particles were further $3 \mathrm{D}$ classified with a transmembrane domain mask in $\mathrm{C} 4$ symmetry, followed by 3D auto-refine and CTF refinement. After that, Bayesian polishing was performed, and bad particles were removed from polishing particles using 2D Classification. The selected polishing particles were subjected to 3D auto-refine in RELION and then Non-uniform refinement in cyroSPARC. The final reconstruction was reported at $3.0 \AA$ for Shaker-IR and 2.9 $\AA$ for W434F. To confirm whether ion density in the selectivity filter is real, the reference was shifted by 10 pixels in X-direction using relion_image_handler so that the selectivity filter is not in the center axis. C1 symmetry was applied for 3D auto-refine. The ion density without postprocessing was shown in Fig.S9.

\section{Model building and structure refinement}

Model building was first carried out by manually fitting the transmembrane domain of Kv1.2-2.1 paddle chimera channel (PDB 6EBM) into the electron microscopy density map using UCSF Chimera (69). The model was then manually built in Coot (70) and refined using real_space refinement in PHENIX (71) with secondary structure and geometry restraints. The final model was evaluated by comprehensive validation in PHENIX. Structural figures were generated using PyMOL (https://pymol.org/2/support.html) and UCSF Chimera.

\section{Structural Alignments}

Alignments of single subunits (Fig. S7) were performed using align in PyMOL. Alignments of tetramers (Fig. S4, S10) were performed using Fr-TM-Align (72). Alignments between KcsA and Shaker (Fig. S10) were performed using the pore domain (S5-S6 helices) of all four subunits. Alignments between the Kv 1.2/2.1 paddle chimera and Shaker (Fig. S4) were performed using the transmembrane domain (S1-S6 helices) of all four subunits. Pore radii were calculated using the HOLE program (73).

\section{Molecular dynamics simulations of Shaker-IR and the W434F mutant}

The simulations of Shaker-IR and the W434F mutant used the cryo-EM structures reported in this study as the starting configuration; in both cases, the simulations examined a construct encompassing residues 382 to 485 (from S4-S5 helix to S6 helix), with neutral N- and C-termini 
and all ionizable sidechains in their default protonation state at $\mathrm{pH} 7$ (the net charge of these constructs is thus zero). For Shaker-IR, two $\mathrm{K}^{+}$ions were initially positioned in the selectivity filter, one coordinated by residues 442 and 443 and another by residues 444 and 445; a third ion was positioned below the sidechain of 442 , and two water molecules were modeled between the three ions. For the W434F mutant, $\mathrm{K}^{+}$ions were initially positioned between residues 443 and 444 and by residue 442, with a water molecule in between. (Note neither configuration was ultimately the most populated in the resulting trajectories.) Dowser (74) was used to model water molecules buried within the protein structures. To complete the initial set-up of the experimental cryo-EM structures, both constructs (including ions and water molecules) were briefly energy-minimized using CHARMM(75) and the CHARMM36m forcefield(76-78); specifically, the minimization consisted of 250 steps using the steepest-descent algorithm, followed by 250 steps using the conjugate-gradient algorithm. Both Shaker-IR and W434F were simulated in a POPC lipid bilayer flanked by a $300 \mathrm{mM} \mathrm{KCl}$ solution. To generate a molecular model of this membrane/solvent environment, we created a coarse-grained (CG) POPC-lipid bilayer in $300 \mathrm{mM} \mathrm{KCl}$ in an orthorhombic box of $\sim 90 \times 90 \times 100 \AA$, using insane.py (79). To equilibrate this CG system, we carried out a 20- $\mu$ s MD simulation using GROMACS 2018.8 (80) and the Martini2.2 forcefield (81-84), at constant temperature (303 K) and constant semi-isotropic pressure (1 atm) and with periodic boundary conditions. The integration time-step was 20 fs. To embed Shaker-IR in this environment, its atomic structure was first coarse-grained using martinize.py $(81,83)$, and overlaid onto the membrane/solvent system in a configuration that seemed plausible, removing all overlapping lipid/water molecules. To optimize the protein/lipid/solvent interfaces in the resulting model, we carried out a 10- $\mu$ s MD simulation of the complete system using GROMACS 2018.8 (80) and Martini2.2 (81-84) at constant temperature (303 K) and constant semi-isotropic pressure (1 atm) and with periodic boundary conditions and an integration time-step of $20 \mathrm{fs}$. Having ascertained the equilibration of the membrane structure and of the position of the protein in the lipid bilayer, the final snapshot of the CG trajectory was transformed into an all-atom representation based on the CHARMM36m forcefield (76-78). To do so, lipid and solvent molecules were back-mapped (85) onto all-atom models; and the CG version of Shaker-IR was replaced with the energy-minimized all-atom construct described above, after optimally superposing the $\mathrm{C} \alpha$ trace of the latter onto that of the former. The resulting all-atom molecular 
system includes $201 \mathrm{POPC}$ lipids, 14,376 water molecules, $79 \mathrm{~K}^{+}$and $79 \mathrm{Cl}^{-}(300 \mathrm{mM} \mathrm{KCl})$, in an orthorhombic box of ca. $90 \times 90 \times 100 \AA$.

To further optimize this all-atom model, the simulation system was first energy-minimized for 5,000 steps using with NAMD $2.13(86,87)$ and the conjugate-gradient algorithm. We then carried out a series of MD simulations wherein structural restraints are applied to the protein and ions/water in the selectivity filter progressively weakened over the course of $\sim 150 \mathrm{~ns}$. The scheme followed in this multi-step equilibration is summarized in Table S2. These simulations were carried out using NAMD $2.13(86,87)$ and CHARMM36m (76-78) at constant temperature (298 K) and constant semi-isotropic pressure (1 atm) with periodic boundary conditions and an integration time-step of 2 fs. Electrostatic interactions were calculated using PME, with a realspace cut-off value of $12 \AA$; van der Waals interactions were also cut-off at $12 \AA$, with a smooth switching function taking effect at $10 \AA$. The all-atom simulation system for the W434F mutant was constructed by replacing the structure of Shaker-IR with the structure of the W434F mutant at the end of Step 6 in the equilibration protocol summarized in Table 2. The resulting all-atom molecular system includes 201 POPC lipids, 14,375 water molecules, $79 \mathrm{~K}^{+}$and $79 \mathrm{Cl}^{-}(300 \mathrm{mM}$ $\mathrm{KCl}$ ), in an orthorhombic box of ca. $90 \times 90 \times 100 \AA$. The complete minimization/equilibration protocol (Steps 1 through 9) was then repeated for the W434F mutant. No external electric field was applied during either the equilibration of Shaker-IR or the W434F mutant, and so the transmembrane voltage in both cases was zero. The dimensions of both simulation systems at the end of the equilibration protocol are ca. $89 \times 89 \times 92 \AA$. Finally, to quantify the ion-conducting properties of Shaker-IR and the W434F mutant, a 3.5- $\mu$ s MD trajectory was calculated for each construct using an Anton 2 supercomputer (88) and the CHARMM36m forcefield (76-78). The starting configuration for each of these simulations was the final configuration after Step 9 in the corresponding equilibration. These simulations were also carried out at constant temperature $(298 \mathrm{~K})$ and semi-isotropic pressure (1 atm), respectively set with the Nose-Hoover thermostat (89, 90) and the Martyna-Tobias-Klein barostat (91), and with periodic boundary conditions and an integration time-step of 2.5 fs. Electrostatic interactions were calculated using the Gaussian split Ewald method (92); van der Waals interactions were cut-off at $10 \AA$. To ensure that the simulations evaluate the properties of the functional states captured by the cryo-EM data, we precluded structural distortions that might occur in the microsecond time-scale by introducing soft restraints on all $\mathrm{F}, \mathrm{Y}$ and $\mathrm{c}_{1}$ dihedral angles. These restraints are identical to those used in previous studies 
based on microsecond simulations calculated on Anton $(93,94)$, but with a smaller force constant equal to $0.6 \mathrm{kcal} / \mathrm{mol}$. The conformational ensembles in these simulations of Shaker-IR and W434F are thus quite diverse; for example, typical RMS deviations of the protein backbone from the cryo-EM structures range from 1 to $1.4 \AA$ (Fig. S9). No external electric field was applied during the first $0.5 \mu \mathrm{s}$ of either of MD trajectories. At $0.5 \mu \mathrm{s}$, a voltage jump to $300 \mathrm{mV}$ was introduced in the system, for both Shaker-IR and the W434F, through the application of an outwardly directed electric field of $0.075 \mathrm{kcal} / \mathrm{mol} \AA^{-1} \mathrm{e}^{-1}$ perpendicular to the membrane plane (1 $\mathrm{kcal} / \mathrm{mol} \AA^{-1} \mathrm{e}^{-1}=43.4 \mathrm{mV} / \AA$ ). For a W434F, a second voltage jump to $450 \mathrm{mV}$ was introduced at $2.5 \mu \mathrm{s}$ through the application of an outwardly directed electric field of $0.1125 \mathrm{kcal} / \mathrm{mol}^{-1} \mathrm{e}^{-1}$. 

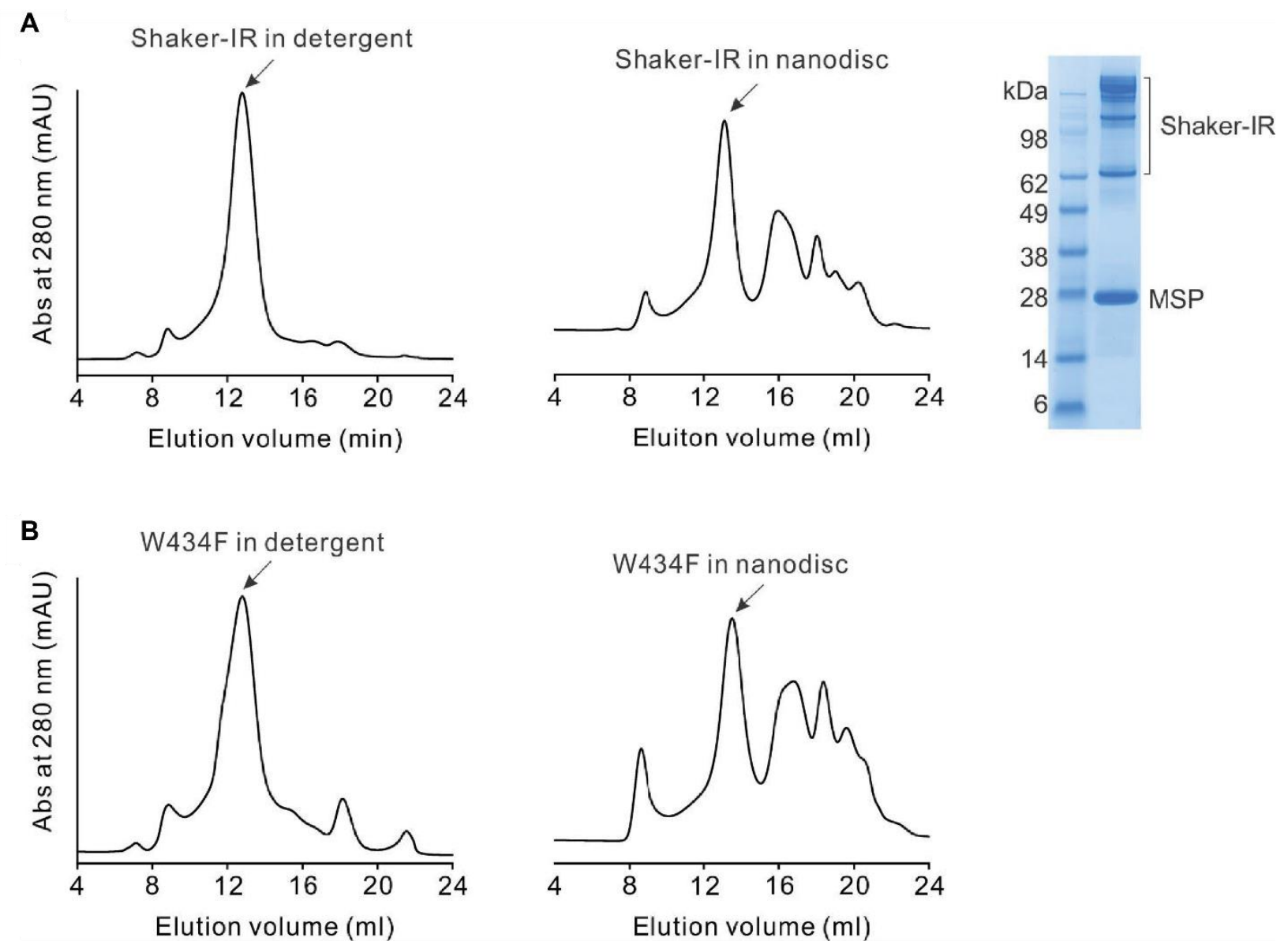

\section{Fig. S1.}

Biochemistry for wild-type Shaker-IR and W434F mutant. (A) Gel filtration chromatograms of the WT Shaker in detergent (left) and in nanodisc (middle), and SDS-PAGE of the WT Shaker in nanodisc. The multiple bands running at a higher molecular weight in the SDS-PAGE appear to reflect the glycosylated species of the channel. (B) Gel filtrationchromatograms of W434F mutant in detergent (left) and in nanodisc (right). The peak fractions marked with the arrow were collected and used either for nanodisc reconstitution or for cryo- EM imaging. 
bioRxiv preprint doi: https://doi.org/10.1101/2021.09.21.461258; this version posted September 21, 2021. The copyright holder for this preprint (which was not certified by peer review) is the author/funder. This article is a US Government work. It is not subject to copyright under 17 USC 105 and is also made available for use under a CCO license.
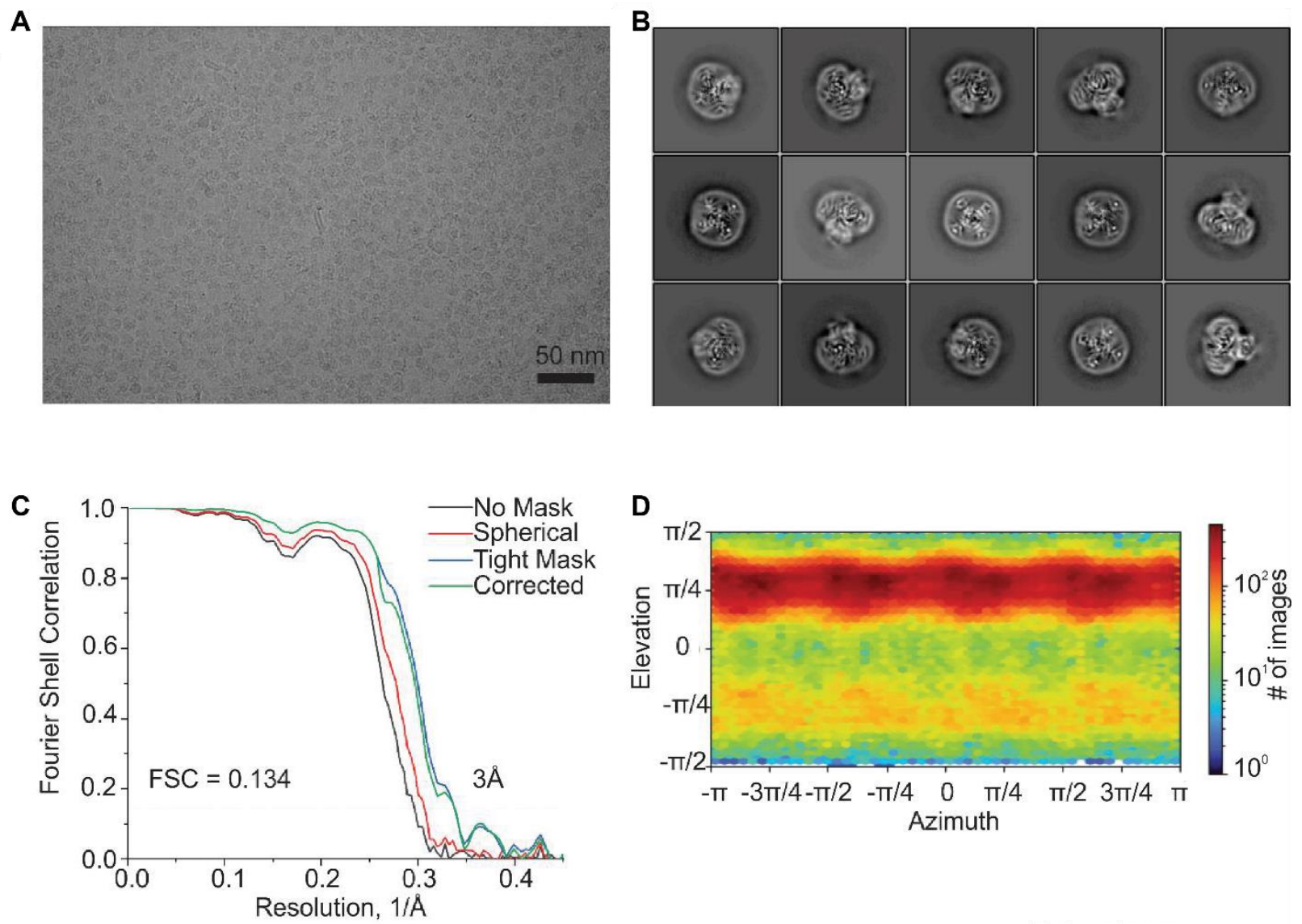

E
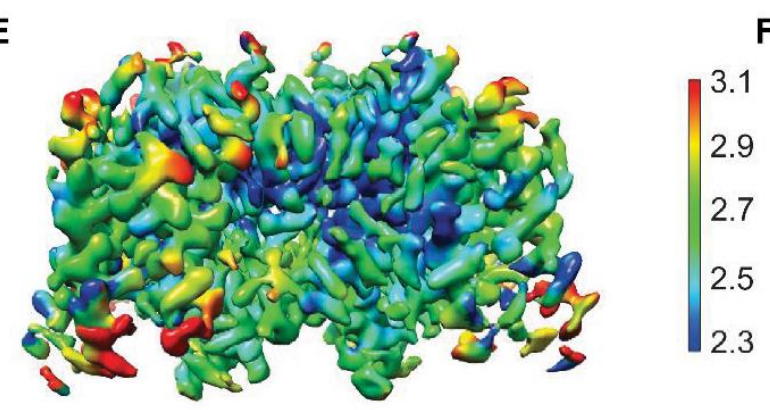

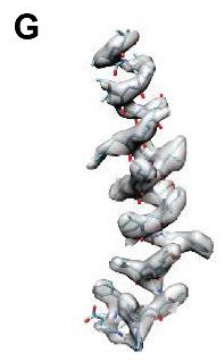

S1

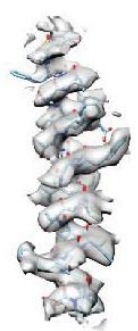

S2

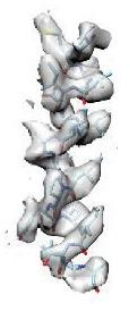

S3

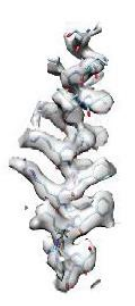

S4
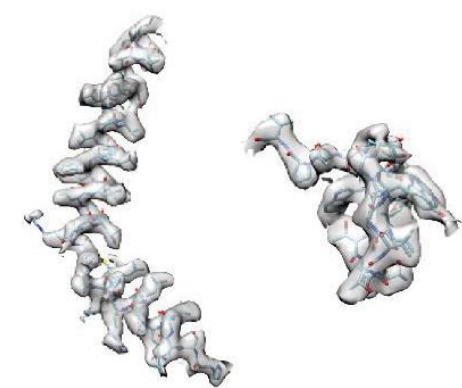

S5_linker
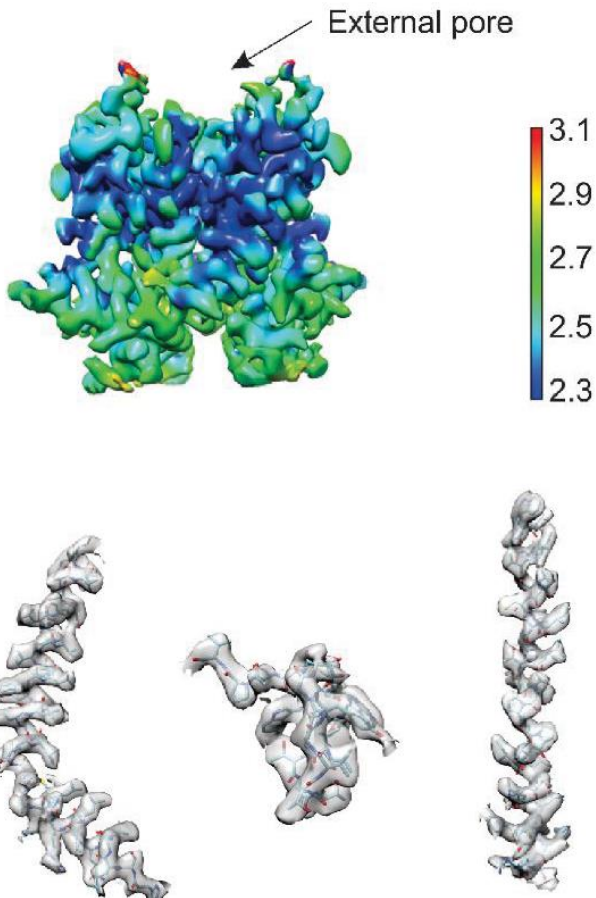

S6 


\section{$\leftarrow$ Fig. S2.}

Cryo-EM imaging of Shaker-IR. (A) Cryo-EM micrograph of Shaker-IR. (B) 2D class averages of the particles in different orientations.(C) Fourier Shell Correlation (FSC) curves. (D) Direction distribution plots of the 3D reconstruction. (E) Local resolution map for the entire TM region. (F) Local resolution map for the S5-S6 pore domain, highlighting the dark blue region within the outer pore that shows the best resolution inthe overall structure. (G) Regional cryo-EM density for Shaker-IR. 
bioRxiv preprint doi: https://doi.org/10.1101/2021.09.21.461258; this version posted September 21, 2021. The copyright holder for this preprint (which was not certified by peer review) is the author/funder. This article is a US Government work. It is not subject to copyright under 17 USC 105 and is also made available for use under a CCO license.
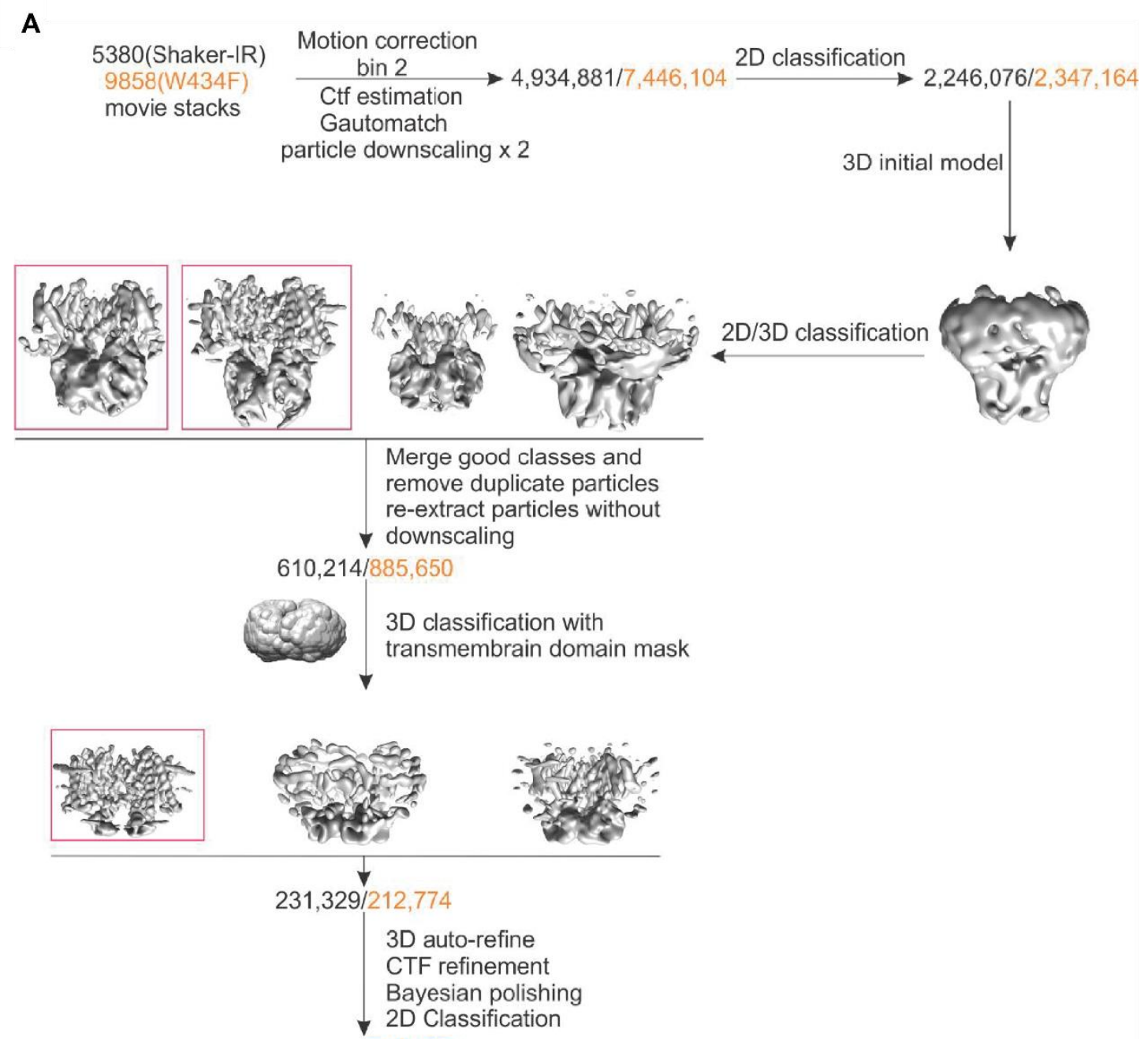

$229,397 / 212,083$

3D auto-refine in relion

Non-uniform refinement

$\checkmark$ in cryosparc

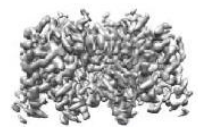

$3.0 \AA / 2.9 \AA$

B
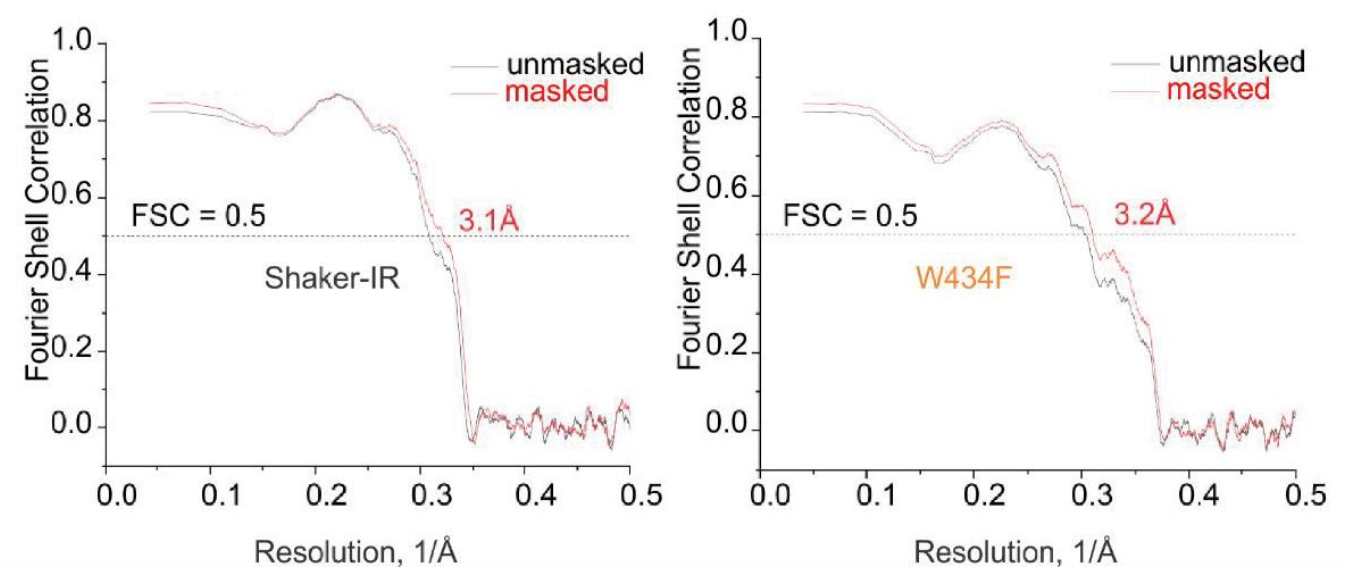
bioRxiv preprint doi: https://doi.org/10.1101/2021.09.21.461258; this version posted September 21, 2021. The copyright holder for this preprint

(which was not certified by peer review) is the author/funder. This article is a US Government work. It is not subject to copyright under 17 USC 105 and is also made available for use under a CCO license.

\section{$\leftarrow$ Fig. S3.}

Data processing workflow for the cryo-EM structures of Shaker-IRand the W434 mutant.

(A) Workflow for the cryo-EM data processing. (B) FSC curves of the model versus map. 
bioRxiv preprint doi: $h$ ttps://doi org/10.1101/2021.09.21.461258; this version posted September 21,2021 . The copyright holder for this preprint

(which was not certified by peer review) is the author/funder. This article is a US Government work. It is not subject to copyright under 17 USC 105 and is also made available for use under a CCO license.

A

Shaker-IR

Kv 1.2/2.1 paddle chimera
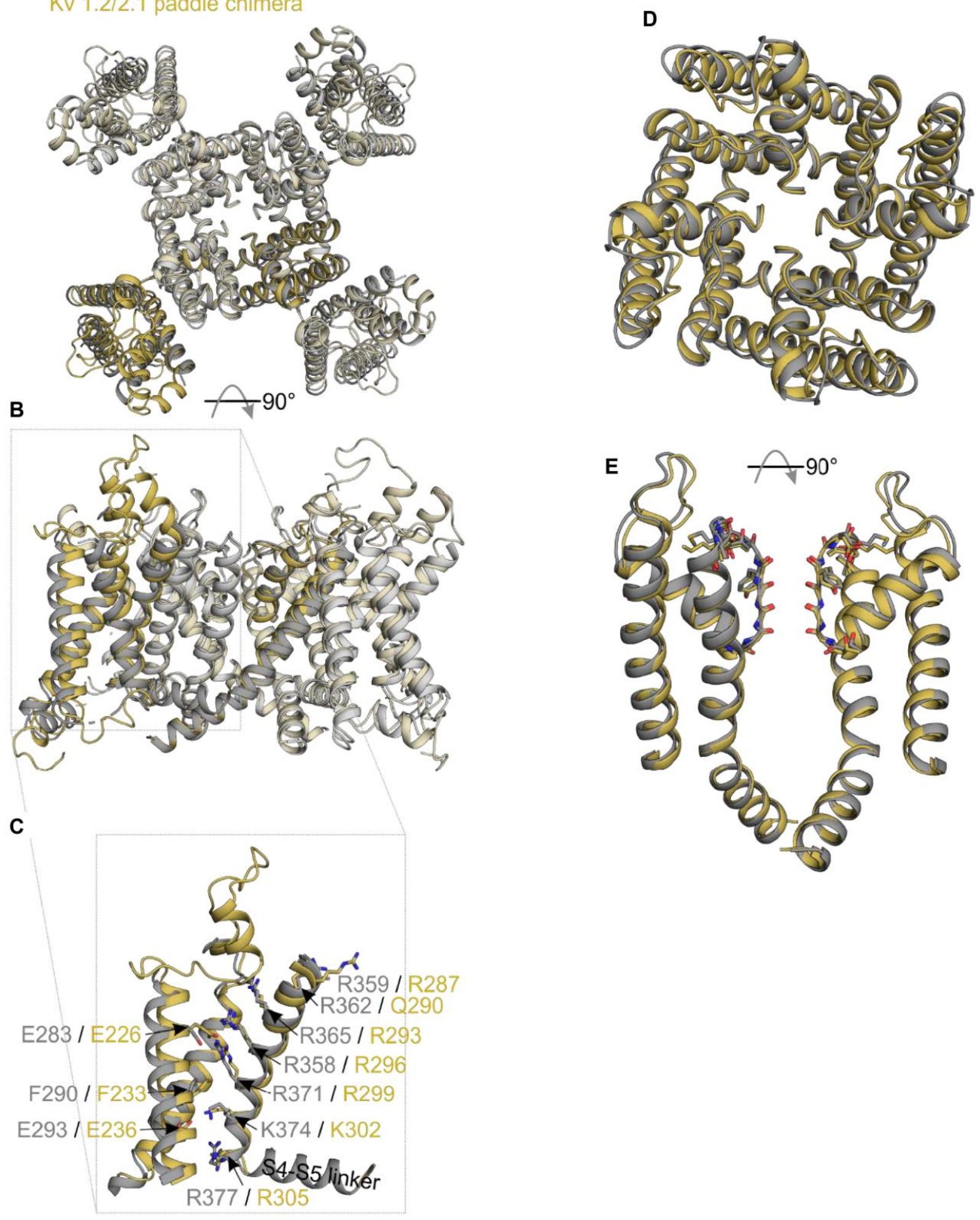


\section{$\leftarrow$ Fig. S4.}

Structural alignment of Shaker $\mathrm{Kv}$ channels with the Kv2.1/1.2 paddle chimera. Superimposition of transmembrane domains (S1-S6) of Shaker-IR (XXXX) and the Kv 1.2/2.1 paddle chimera (2r9r) (A) top and (B) side views, along with (C) focused view of one S1-S4 voltage- sensing domain with S4 charges and charge transfer center residue side chains shown as sticks. Superimposition of pore domains (S5-S6) (D) top and (E) side views. Superimpositions of the transmembrane domains (S1-S6) were created using Fr-TM-Align. 
bioRxiv preprint doi: https://doi.org/10.1101/2021.09.21.461258; this version posted September 21, 2021. The copyright holder for this preprint (which was not certified by peer review) is the author/funder. This article is a US Government work. It is not subject to copyright under 17 USC 105 and is also made available for use under a CCO license.

Shaken'1-655 Kv1.2/1-499 Kv2.1/1-857 Kv1.2/2.1/1-513 Kv3.1/1-585 Kv4.1/1-651

Shaken'1-655 Kv1.2/1-499 Kv2.1/1-857 Kv1.2/2.1/1-513 Kv3.1/1-585 Kv4.1/1-651 (

Shaken'1-655 Kv1.2/1-499 Kv2.1/1-857 Kv1.2/2.1/1-513 Kv3.1/1-585 Kv4.1/1-651

KcsAv1-160 Shaker/1-655 Kv1.2/1-499 Kv2.1/1-857 Kv1.2/2.1/1-513 Kv3.1/1-585 Kv4.1/1-651

\section{KesAV1-160} Shaken'1-655 Kv1.2/1-499 Kv2.1/1-857 Kv1.2/2.1/1-513 Kv3.1/1-585 Kv4.1/1-651

KCSAV1-160 Shaken'1-655 Kv1.2/1-499 Kv2.1/1-857 Kv1.2/2.1/1-513 Kv3.1/1-585 Kv4.1/1-651
194 EGF IKEE . . . E . . . . . . . . RPLP. . DNEKQRKVWLLFEYPESSQAARVVA II 232 130 EGYIKEE . . . . . . . . . . . RPLP. . ENEFQRQVWLLFEYPESSGPARIIA IV 168 152 AETLREREGEEF........... DNTC... CAEKRKKLWDLLEKPNSSVAAKILAII 194 148 EGYIKEE .... . . . . . . . . RPLP. . ENEFQRQVWLLFEYPESSGPARIIAIV 186 137 DGPGDSGDGEDELEMTKRLALSDSPDGRPGGFWRRWQPR I WALFEDPYSSRYARYVAFA 195 151 EEAEQAGEG.................... SSLRQRLWRAFENPHTSTAALVFYYV 191

S2

233 SVFVILLSIVIF CLETLPEFKHYKVFN...... TTTNGTKIEEDEVPDITDPFFLIET 284 169 SVMVILISI VSF CLETLP I FRDENEDMHGGGVTFHTYSNSTIGYQQSTSFTDPFF IVET 227 195 SIMF I VLSTIALSLNTLPELQSLDEFGQST............................. 187 SVMV I L I S I VSF CLETLP I FRDENEDMHGGGVTFHTYSQST I GYQQSTSFTDPFF I VET 245 196 SLFF I LVS I T TF CLETHERFNP I VNKTE IE. . . NVRNG TQVRYYREAETEAFLTYIEG 250 192 TGFF I AVSVIANVVETI PCRGTPRWPS..........................

\section{S3}

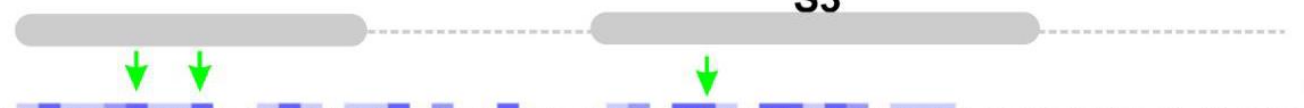

285 LC I I WF TFELTVRFLACPNKLNF CRDVMNVI D I I A I I PYF I TLATVVAEEEDTLNLPKA 343 228 LC I I WF SFEFLVRFFACPSKAGFF TN I MN I I D I VA I I PYF I TLGTELAEKPE..... 279 235 VC I AWF TMEYLLRFLSSPKKWKF F KGPLNA I DLLA I LPYYVT IFLTESNKSV . . . . 286 246 LCI I WF SFEF LVRFFACPSKAGFFTN I MN I I D I VA I I PYYVT I FLTESNKSV..... 297 251 VCVVWF TFEF LMRVVF CPNKVEF I KNSLN I I DFVA I LPFYLEVGLSGLSSK...... 301 237 ACVL I F TGEYLLRLFAAPSRCRFLRSVMSLI DVVAILPYYI GLFVPKNDDV . . . . . 287 S4

$1 \ldots . .$. MPPML SGLLA... . . . LVKLLLGRHGSALH....WRAAGAATVLLVIV 39 344 PVSPQDKSSNQAMSLA I LRV I RLVRVFR I F KLSRHSKGLQ ILGRTLKASMRELGLL IFF 402 280 ... DAQQGQQAMSLA ILRVIRLVRVFR I F KLSRHSKGLQ ILGQTLKASMRELGLLIFF 334 $287 \ldots$... LQF... Q QNVRRVVQ IFR I MR I LR I LKLARHSTGLQSLGF TLRRSYNELGLL ILF 337 298 ... LQF... Q QNVRRVVQ IFR I MR I LR I F KLSRHSKGLQ ILGQTLKASMRELGLL IFF 348 $302 \ldots . . . . . A A K D V L G F L R V V R F V R$ I LR I F KLTRHFVGLRVLGHTLRASTNEFLLL I I F 351 $288 \ldots \ldots \ldots . . . . .6$ SGFVTLRVFRVFR IF KF SRHSQGLRILGYTLKSCASELGFLLFS 332 pore helix

40 LLAG... SYLAVLAER ........ GAPGAQL I TYPRAL WWSVETATTVGYGDLYPVT 85 403 LF I GVVLFSSAVYFAEA........ GSENSFFKSI PDAFWWAVVTMT TVGYGDMTPVG 452 335 LF I GVILFSSAVYFAEA........ DERDSQFPS I PDAF WWAVVSMT TVGYGDMVPTT 384 338 LAMG IMIF SSLVFFAEK........ DEDDTKF KS I PASF WWAT I TMT TVGYGDIYPKT 387 349 LF I GVILFSSAVYFAEA....... DERDSQFPS I PDAFWWAVVSMT TVGYGDMVPT T 398 352 LALGVL I FATMIYYAER I GAQPNDPSASEHTHF KN I P I GF WWAVVTMT TLGYGDMYPQT 410 333 LTMA I I I FA TVMF YAEK......... T S KTNF TSI PAAFWYT I VTMT TLGYGDMVPST 382 S6

86 LWGRLVAVVVMVAGITSFGLVTAALATWFV...GREQERRG . . . . . HFVRHSEKAAE 134 453 VWGK I VGSLCA I AGVLT I ALPVPVIVSNF NYF YHRETDQEEMQSQNFNHVTSCP. YLPG 510 385 I GGK I VGSLCA I AGVLT I ALPVPVIVSNF NYF YHRETEGEEQ. A AQYLQVTSCP. KIPS 440 388 LLGK I VGGL C C I AGVLVI ALP I P I I VNNF SEF YKEQKRQEKA I KRREA. LERA - KRNG 443 399 I GGKI VGSLCA I AGVLT I ALPVPVI VSNFNYFYHRETEGEEQ. AQYLQVTSCP. KIPS 454 411 WSGMLVGAL CALAG VLT I AMPVPVI VNNFGMYYSLAMAKQKLPKKKKKH I PRPP. QLGS 468 383 I AGKIFGS I CSLSGVLVIALPVPVIVSNFSR I YHQNQRADKRRAQQKVRLARIR. LAKS 440 


\section{$\leftarrow$ Fig. S5.}

Sequence alignment of $\mathbf{K v}$ channels. Sequence alignment of Shaker (Drosophila melanogaster P08510), KcsA (Streptomyces lividans P0A334), Kv1.2 (Rattus norvegicus P63142), Kv2.1 (Rattus norvegicus P15387), Kv1.2/2.1 chimera(Rattus norvegicus PDB: 6EBK), Kv3.1 (Rattus norvegicus $\mathrm{P} 25122$ ) and Kv4.1 (Mus Musculus $\mathrm{Q} 03719$ ). Blue regions indicate similarity and dark blue region indicate identity. Cartoons represent secondary structure features. Green arrows highlight important residues in the chargetransfer center and red bar indicates residues that change conformation most dramatically between Shaker-IR and W434F. 
bioRxiv preprint doi: https://doi.org/10.1101/2021.09.21.461258; this version posted September 21, 2021. The copyright holder for this preprint (which was not certified by peer review) is the author/funder. This article is a US Government work. It is not subject to copyright under 17 USC 105 and is also made available for use under a CCO license.
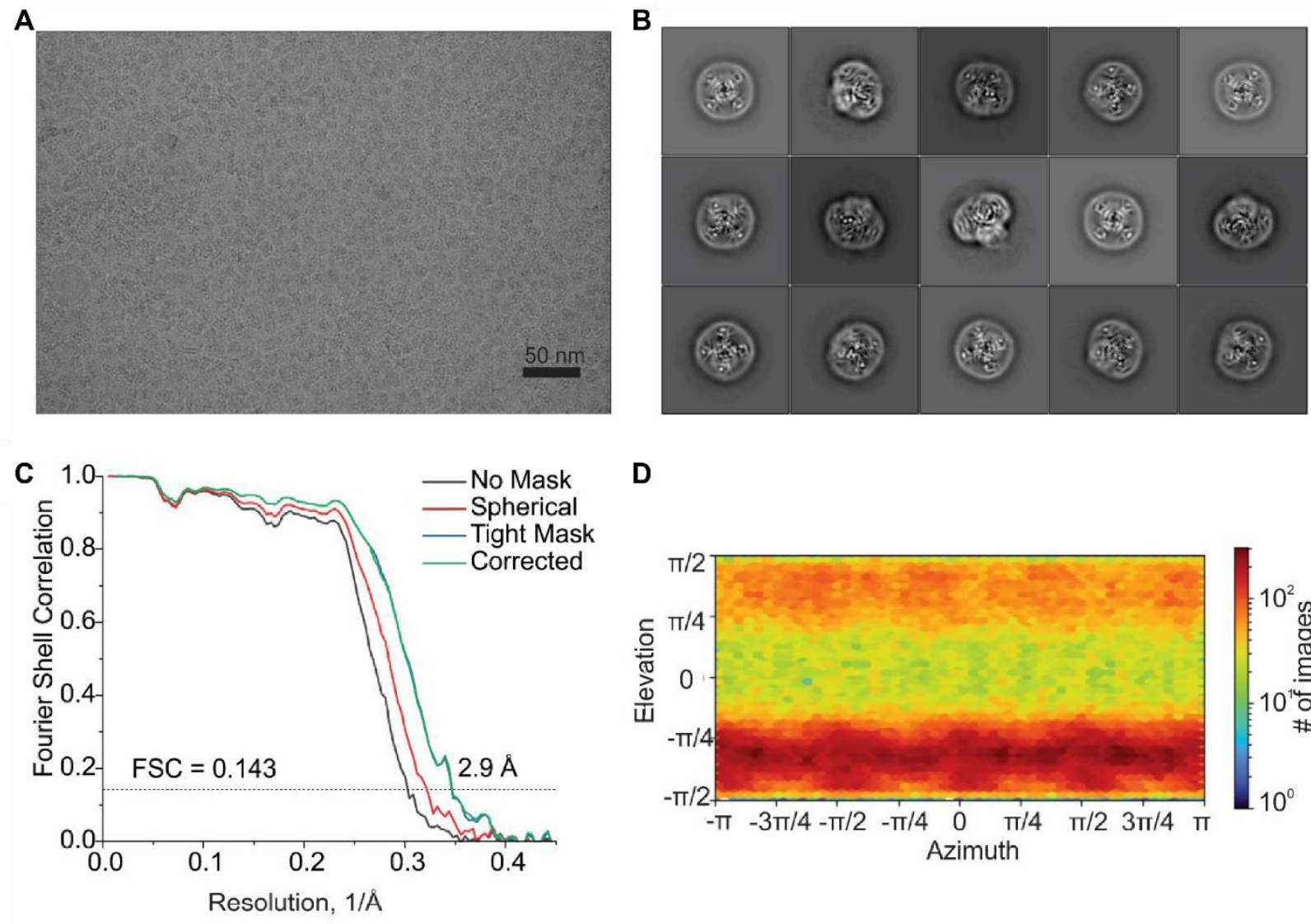

D

E

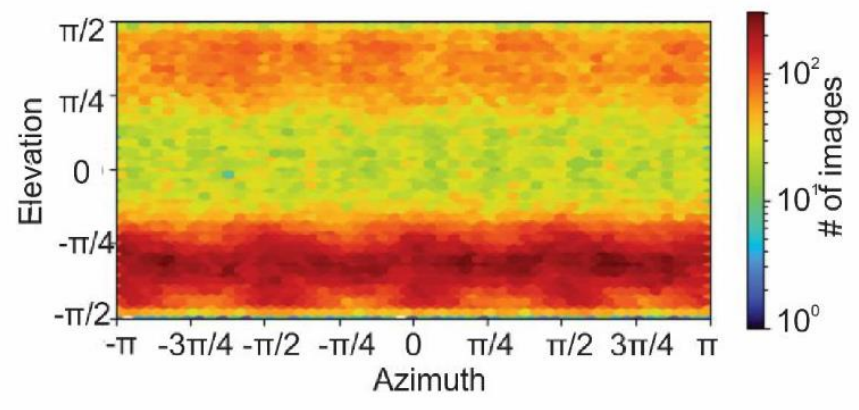

$\mathbf{F}$
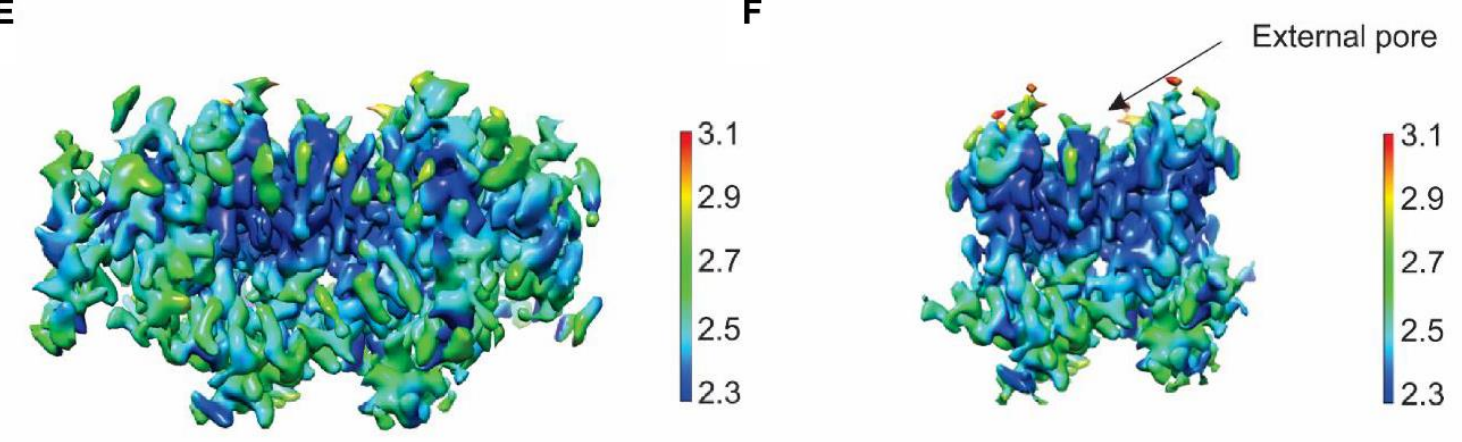

G

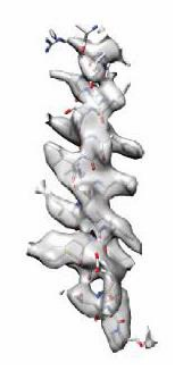

S1

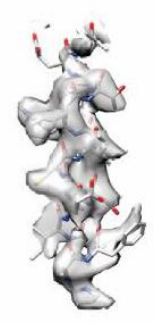

S2

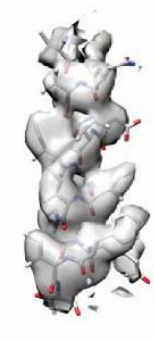

S3

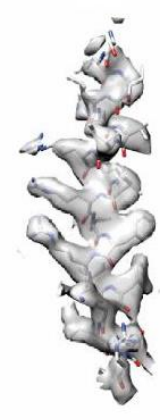

S4

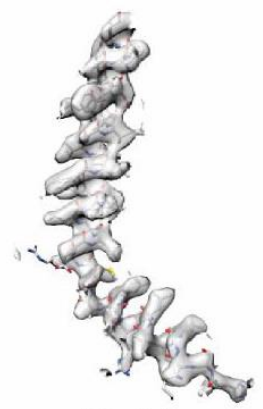

S5_linker

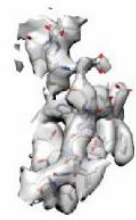

P-loop

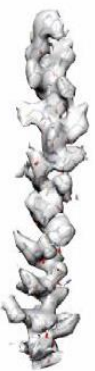

S6 


\section{$\leftarrow$ Fig. S6.}

Cryo-EM imaging of Shaker-IR W434F. (A) Cryo-EM micrograph of Shaker-IR W434F. (B) 2D class averages of the particles in different orientations. (C) Fourier Shell Correlation (FSC) curves. (D) Direction distribution plots of the 3D reconstruction. (E) Local resolution map for the entire TM region. (F) Local resolution map for the S5-S6 pore domain, highlighting the dark blue region within the outer pore that shows the best resolution in the overall structure. (G) Regional cryo-EM density for Shaker-IR W434F. 
A
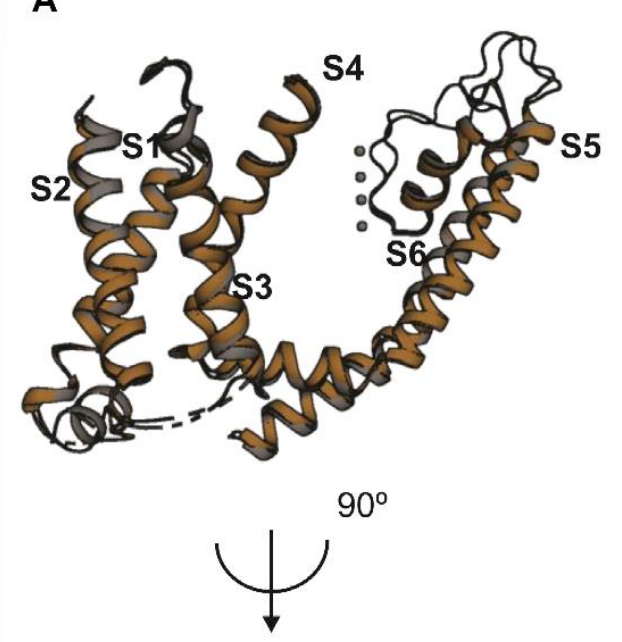

Fig. S7.

Structural alignment of Shaker-IR with the W434F mutant. (A) Superimposition of the Shaker-IR (gray) and W434F (brown) viewed from the side (top image)or from the external side of the membrane (bottom). (B) Close-up view of the conformational change within the outer pore domain viewed from the external side of the membrane. (C) cryo- EM density of P-loop for the W434F mutant. Density for two distinct rotamers of D447 are indicated by arrows. 
A

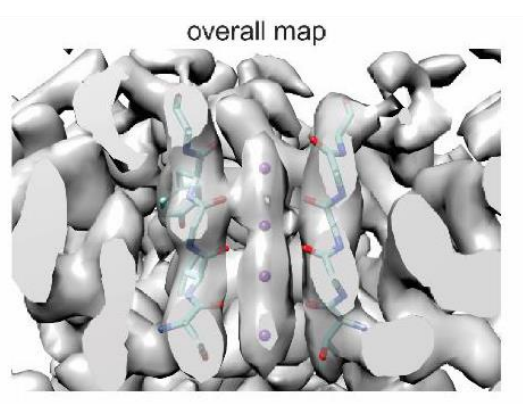

B

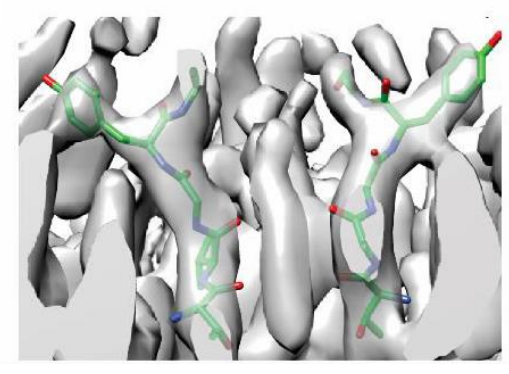

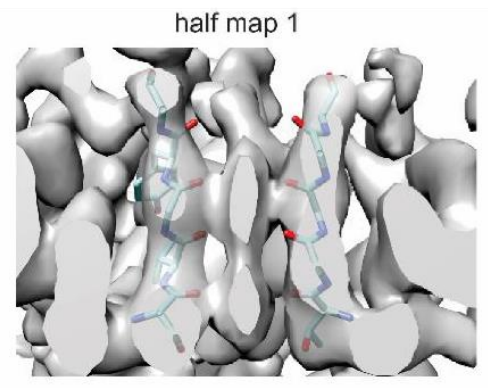
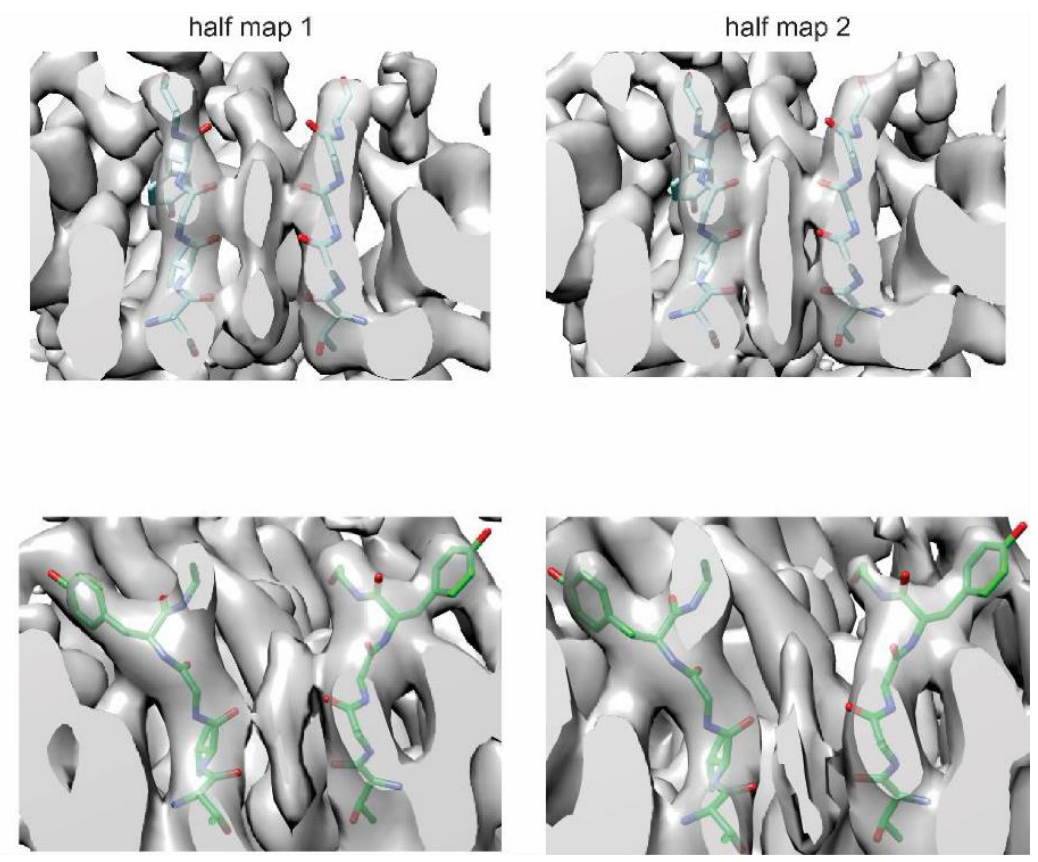

\section{Fig. S8.}

\section{Ion densities in the selectivity filter of the wild-type Shaker-IR channeland W434F mutant.}

(A) Ion density of Shaker-IR using C1 symmetry reconstruction shown in the overall map and half maps (lowpass at $3.5 \AA$ A). 4 ions (purple) are fit into S1-S4 sites (left panel), with residues in the filter shown in stick. (B) Ion density of Shaker-IR W434F using C1 symmetry reconstruction shownin the overall map and half maps (lowpass at $3.5 \AA$ ). Cryo-EM density between S1 and S2 sites inW434F are much weaker compared the Shaker-IR. 

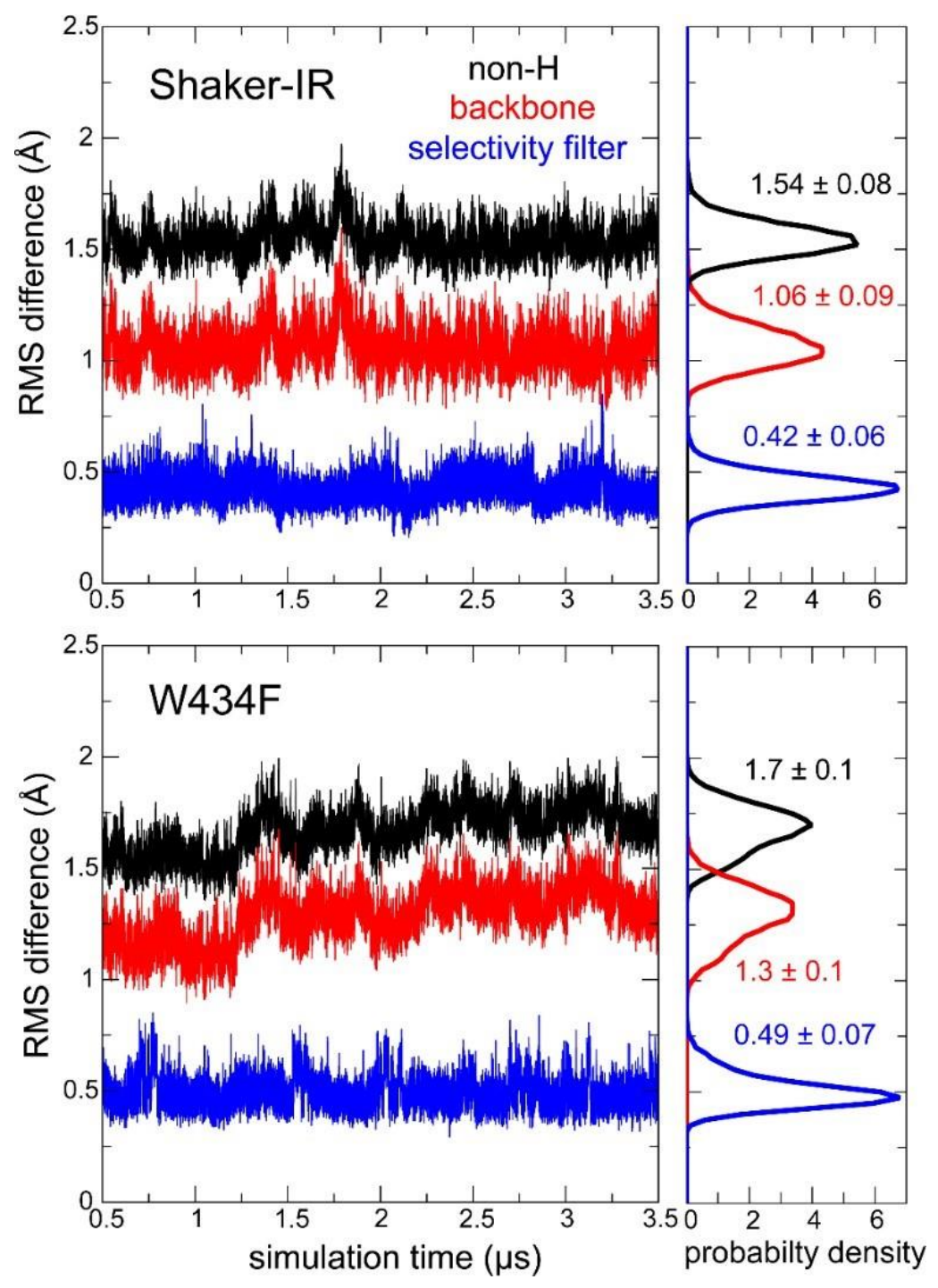

Fig. S9.

MD simulations of Shaker-IR and the W434F mutant. For the portion of the calculated MD trajectories wherein a transmembrane voltage is applied $(300 \mathrm{mV}$ for Shaker-IR and 300/450 mV for W434F), the figures on the left side quantify the root-mean-square (RMS) difference between each of the snapshots (in intervals of $120 \mathrm{ps}$ ) and the corresponding cryo-EM structure. RMS differences are quantified for all non-hydrogen atoms in the protein structures (black), only for the backbone atoms (red), and only for the backbone atoms of residues 442 to 445 . On the right, the figure shows normalized probability histograms of the time-series shown on the left; mean values and standard-deviations are provided in each case. 

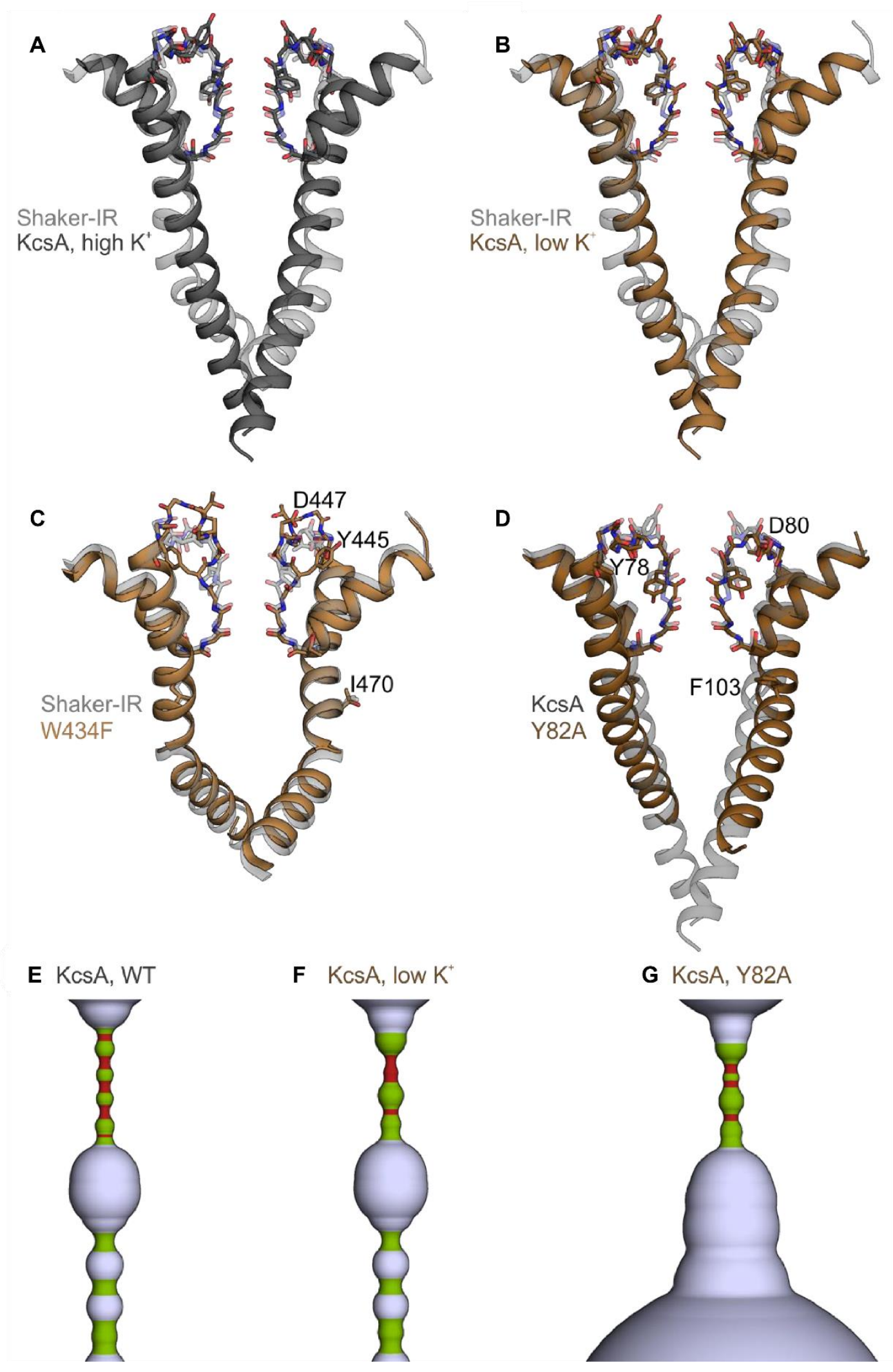

F KcsA, low $\mathrm{K}^{+}$

G KcsA, Y82A
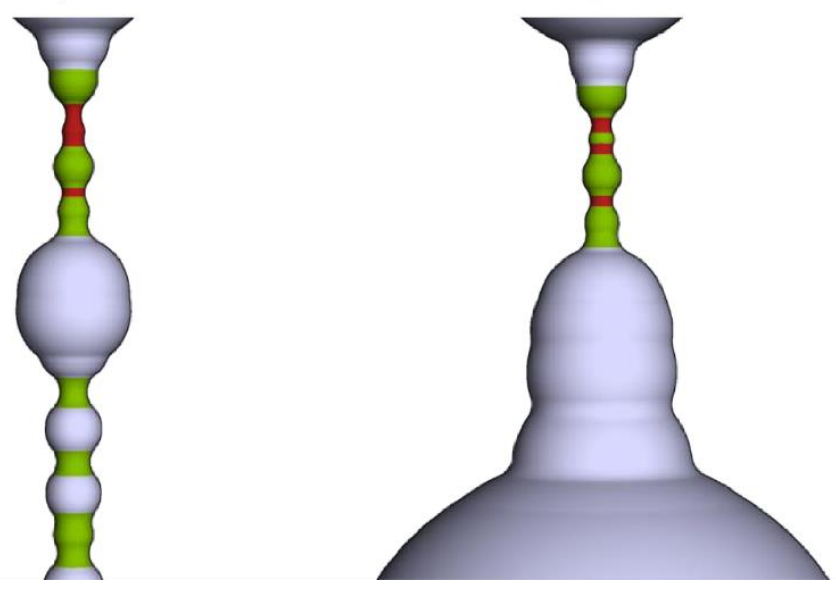


\section{$\leftarrow$ Fig. S10.}

Comparison of Shaker Kv channel structures to KesA. (A) Superimposition of S6 and pore helix (two opposing subunits) of Shaker-IR (XXXX) and KcsA inthe presence of high $\mathrm{K}^{+}(200$ $\left.\mathrm{mM} \mathrm{K}^{+}, 1 \mathrm{k} 4 \mathrm{c}\right)$. (B) Superimposition of S6 and pore helix of Shaker-IR (XXXX) and KcsA in the presence of low $\mathrm{K}^{+}\left(3 \mathrm{mM} \mathrm{K}^{+}, 1 \mathrm{k} 4 \mathrm{~d}\right)$. (C) Superimposition of $\mathrm{S} 6$ and pore helix of Shaker-IR (XXXX) and W434F mutant (XXXX). (D) Superimposition of S6 and pore helix of KcsA in the presence of high $\mathrm{K}^{+}(1 \mathrm{k} 4 \mathrm{c})$ and $\mathrm{KcsA}$ fast-inactivating Y28A mutant in the inactivated/open state (5vke). Superimpositions of the pore domain (S5-S6) were created using Fr-TM-Align. HOLE diagrams of (E) KcsA in the presence of high $\mathrm{K}^{+}(1 \mathrm{k} 4 \mathrm{c}),(\mathbf{F}) \mathrm{KcsA}$ in the presence of low $\mathrm{K}^{+}$ (1k4d), and (G) KcsA fast-inactivating Y28K mutant (5vke). Radii $\leq 1 \AA$ are shown in red, radii $\leq 2 \AA$ and $>1 \AA$ are shown in green, and radii larger than $2 \AA$ are shown in light blue. 


\section{Table S1.}

Cryo-EM data collection, refinement and validation statistics.

\begin{tabular}{|c|c|c|}
\hline & $\begin{array}{l}\text { Shaker-IR } \\
(\text { EMDB-xxxx) } \\
(\text { PDB xxxx) }\end{array}$ & $\begin{array}{l}\text { Shaker- } \\
\text { W434F } \\
(\text { EMDB-xxxx) } \\
(\text { PDB xxxx) }\end{array}$ \\
\hline \multicolumn{3}{|l|}{$\begin{array}{l}\text { Data collection and } \\
\text { processing }\end{array}$} \\
\hline Magnification & 105,000 & 105,000 \\
\hline Voltage (kV) & 300 & 300 \\
\hline Detector & Gatan K3 & Gatan K3 \\
\hline Electron exposure $\left(\mathrm{e}-/ \AA^{2}\right)$ & 52 & 52 \\
\hline Defocus range $(\mu \mathrm{m})$ & -0.5 to -1.5 & -0.5 to -1.5 \\
\hline Pixel size $(\AA)$ & 0.43 & 0.43 \\
\hline Symmetry imposed & $\mathrm{C} 4$ & $\mathrm{C} 4$ \\
\hline Initial particle images (no.) & $4,934,881$ & $7,446,104$ \\
\hline Final particle images (no.) & 229,379 & 212,083 \\
\hline Map resolution $(\AA)$ & 3.0 & 2.9 \\
\hline FSC threshold & 0.143 & 0.143 \\
\hline \multicolumn{3}{|l|}{ Refinement } \\
\hline $\begin{array}{l}\text { Initial model used (PDB } \\
\text { code) }\end{array}$ & $6 \mathrm{ebm}$ & $6 \mathrm{ebm}$ \\
\hline Model resolution $(\AA)$ & 3.1 & 3.2 \\
\hline FSC threshold & 0.5 & 0.5 \\
\hline Map sharpening $B$ factor $\left(\AA^{2}\right)$ & -129 & -102 \\
\hline \multicolumn{3}{|l|}{ Model composition } \\
\hline Non-hydrogen atoms & 7560 & 6495 \\
\hline Protein residues & 892 & 784 \\
\hline Ligands & 36 & 35 \\
\hline \multicolumn{3}{|l|}{$B$ factors $\left(\AA^{2}\right)$} \\
\hline Protein & 65.23 & 35.3 \\
\hline Ligand & 60.7 & 28.7 \\
\hline \multicolumn{3}{|l|}{ R.m.s. deviations } \\
\hline Bond lengths ( $\mathrm{A})$ & 0.003 & 0.003 \\
\hline Bond angles $\left({ }^{\circ}\right)$ & 0.508 & 0.531 \\
\hline \multicolumn{3}{|l|}{ Validation } \\
\hline MolProbity score & 1.14 & 1.43 \\
\hline Clashscore & 3.49 & 7.17 \\
\hline Poor rotamers $(\%)$ & 0 & 0 \\
\hline \multicolumn{3}{|l|}{ Ramachandran plot } \\
\hline Favored $(\%)$ & 98.72 & 98.01 \\
\hline Allowed (\%) & 1.28 & 1.99 \\
\hline Disallowed (\%) & 0 & 0 \\
\hline
\end{tabular}




\section{Table S2.}

Multistep procedure used equilibrate the molecular systems constructed in this simulation study.

\begin{tabular}{|c|c|c|c|c|c|c|c|c|c|}
\hline Step & 1 & 2 & 3 & 4 & 5 & 6 & 7 & 8 & 9 \\
\hline $\begin{array}{l}\text { Positional restraints }{ }^{a} \\
\left(\mathrm{kcal} / \mathrm{mol} / \AA^{2}\right)\end{array}$ & 60 & 60 & 15 & 4 & - & - & - & - & - \\
\hline $\begin{array}{l}\text { Distance restraints }{ }^{b} \\
\left(\mathrm{kcal} / \mathrm{mol} / \AA^{2}\right)\end{array}$ & - & - & - & - & 4 & 1 & 1 & - & - \\
\hline $\begin{array}{c}\text { Dihedral } \Phi, \Psi \text { and } \chi_{1} \text { restraints } \\
\left(\mathrm{kcal} / \mathrm{mol} / \mathrm{deg}^{2}\right)\end{array}$ & - & - & - & - & 16 & 4 & 4 & 4 & 1 \\
\hline $\begin{array}{c}\text { Distance-to-center restraints }{ }^{\mathrm{c}} \\
\left(\mathrm{kcal} / \mathrm{mol} / \AA^{2}\right)\end{array}$ & - & - & - & - & 60 & 15 & - & - & - \\
\hline $\begin{array}{c}\text { Center-of-mass restraints }{ }^{d} \\
\left(\mathrm{kcal} / \mathrm{mol}^{\prime} \AA^{2}\right)\end{array}$ & 1 & 1 & 1 & 1 & 1 & 1 & 1 & 1 & 1 \\
\hline Length of simulation (ns) & 1 & 1 & 1 & 1 & 50 & 25 & 25 & 25 & 25 \\
\hline
\end{tabular}

${ }^{\text {a }}$ Positional restraints on all non-hydrogen atoms in the protein and on $\mathrm{K}^{+}$and water oxygens in the selectivity filter

b Distance restraints on selected hydrogen-bonds and hydrophobic interactions behind the selectivity filter

${ }^{\mathrm{c}} \mathrm{K}^{+}$and water molecules were restrained to remain near the geometric center of their designated binding sites in selectivity filter; each center was defined by eight backbone/sidechain oxygens

${ }^{d}$ The protein center-of-mass was restrained to remain near the center of the simulation box 


\section{Movie S1.}

Morph between the structures of Shaker-IR and the W434F mutant

\section{Movie S2.}

MD simulation of $\mathbf{K}^{+}$dynamics and permeation through Shaker-IR. The movie depicts snapshots of $\mathrm{K}^{+}$(magenta, red, purple, yellow, orange spheres) near and withinthe selectivity filter of Shaker-IR (gray cartoon), for a $1-\mu \mathrm{s}$ fragment of the trajectory (starting at $1.5 \mu \mathrm{s}$ ), under $300 \mathrm{mV}$. Water molecules with $3.8 \AA$ of these $\mathrm{K}^{+}$ions are shown (cyan spheres). The backbone atoms of residues 443-445 and the backbone and sidechain atoms of residue 442are highlighted (excluding hydrogens).

\section{Movie S3.}

MD simulation of $\mathrm{K}^{+}$dynamics and permeation through Shaker-W434F. The movie depicts snapshots of $\mathrm{K}^{+}$(magenta, red, purple, yellow, orange spheres) near and withinthe selectivity filter of Shaker-W434F (gray cartoon), for a 1- $\mu$ s fragment of the trajectory (starting at $2.0 \mu \mathrm{s}$ ), under $300 \mathrm{mV}$ for the first $500 \mathrm{~ns}$ and under $450 \mathrm{mV}$ for the last $500 \mathrm{~ns}$. Watermolecules with $3.8 \AA$ of these $\mathrm{K}^{+}$ions are shown (cyan spheres). The backbone atoms of residues 443-445 and the backbone and sidechain atoms of residue 442 are highlighted (excluding hydrogens). 